\title{
Loss of CEP162 function at the primary cilium delays ciliogenesis and causes retinal ciliopathy in humans
}

Nafisa Nuzhat ${ }^{1}$, Kristof Van Schil2,3, Sandra Liakopoulos ${ }^{4}$, Miriam Bauwens ${ }^{2,3}$, Alfredo Duenas Rey $^{2,3}$, Stephan Käseberg ${ }^{5}$, Melanie Jäger ${ }^{6,7}$, Jason R. Willer ${ }^{8}$, Jennifer Winter ${ }^{5}$, Hanh Truong ${ }^{9}$, Nuria Gruartmoner ${ }^{2,3}$, Mattias Van Heetvelde ${ }^{2,3}$, Joachim Wolf ${ }^{10}$, Robert Merget ${ }^{10}$, Sabine Grasshoff-Derr $^{11}$, Jo Van Dorpe ${ }^{12,13}$, Anne Hoorens ${ }^{12,13}$, Heidi Stöhr ${ }^{14}$, Luke Mansard ${ }^{15}$, AnneFrançoise Roux ${ }^{15}$, Thomas Langmann ${ }^{16}$, Katharina Dannhausen ${ }^{16}$, David Rosenkranz ${ }^{17}$, Karl Martin Wissing ${ }^{18}$, Michel Van Lint ${ }^{19}$, Heidi Rossmann ${ }^{20}$, Friederike Häuser ${ }^{20}$, Peter Nürnberg ${ }^{21,22}$, Holger Thiele ${ }^{21}$, Ulrich Zechner ${ }^{5,17}$, Jillian N. Pearring ${ }^{1,8,9 *}$, Elfride De Baere ${ }^{2,3 *}$, Hanno J. Bolz ${ }^{17,23 *}$

${ }^{1}$ Department of Cell and Developmental Biology, University of Michigan, Ann Arbor, MI, U.S.A. ${ }^{2}$ Department of Biomolecular Medicine, Ghent University, Belgium.

${ }^{3}$ Center for Medical Genetics, Ghent University Hospital, Belgium.

${ }^{4}$ Cologne Image Reading Center, Department of Ophthalmology, University Hospital of Cologne, Cologne, Germany

${ }^{5}$ Institute of Human Genetics, University Medical Center Mainz, Mainz, Germany

${ }^{6}$ Department of Ophthalmology, Justus-Liebig-University Giessen, Giessen, Germany

${ }^{7}$ Augenarztpraxis Bad Brückenau, Bad Brückenau, Germany

${ }^{8}$ Department of Ophthalmology, University of Michigan, Ann Arbor, MI, U.S.A.

${ }^{9} \mathrm{Cell}$ and Molecular Biology Program, University of Michigan, Ann Arbor, MI, U.S.A.

${ }^{10}$ Department of Radiology, Bürgerhospital, Frankfurt am Main, Germany

${ }^{11}$ Department of Pediatric Surgery, Bürgerhospital, Frankfurt am Main, Germany

${ }^{12}$ Department of Diagnostic Sciences, Ghent University, Belgium.

${ }^{13}$ Department of Pathology, Ghent University Hospital, Belgium.

${ }^{14}$ Institute of Human Genetics, University of Regensburg, Regensburg, Germany

${ }^{15}$ Laboratoire de Génétique Moléculaire, $\mathrm{CHU}$ de Montpellier, Université de Montpellier, Montpellier, France 
${ }^{16}$ Laboratory for Experimental Immunology of the Eye, Department of Ophthalmology, Faculty of Medicine and University Hospital Cologne, Cologne, Germany

${ }^{17}$ Senckenberg Centre for Human Genetics, Frankfurt am Main, Germany

${ }^{18}$ Department of Nephrology, Brussels University Hospital, Belgium

${ }^{19}$ Department of Ophthalmology, Brussels University Hospital, Belgium

${ }^{20}$ Institute of Clinical Chemistry and Laboratory Medicine, University Medical Center Mainz, Mainz, Germany.

${ }^{21}$ Cologne Center for Genomics (CCG), University of Cologne, Faculty of Medicine and University Hospital Cologne, Cologne, Germany

${ }^{22}$ Center for Molecular Medicine Cologne, University of Cologne, Cologne, Germany

${ }^{23}$ Institute of Human Genetics, University Hospital of Cologne, Cologne, Germany

*These authors contributed equally to this work. 


\section{ABSTRACT}

Ciliopathies often comprise retinal degeneration since the photoreceptor's outer segment is an adapted primary cilium. CEP162 is a distal end centriolar protein required for proper transition zone assembly during ciliogenesis and whose loss causes ciliopathy in zebrafish. CEP162 has so far not been implicated in human disease. Here, we identified a homozygous CEP162 frameshift variant, c.1935dupA (p. $\left.\left(E 646 R^{\star} 5\right)\right)$, in retinitis pigmentosa patients from two unrelated Moroccan families, likely representing a founder allele. We found that even though mRNA levels were reduced, the truncated CEP162-E646R ${ }^{\star} 5$ protein was expressed and localized to the mitotic spindle during mitosis, but not at the basal body of the cilium. In CEP162 knockdown cells, expression of the truncated CEP162-E646R ${ }^{\star} 5$ protein is unable to restore ciliation indicating its loss of function at the cilium. In patient fibroblasts, cilia overcome the absence of CEP162 from the primary cilium by delaying ciliogenesis through the persistence of CP110 at the mother centriole. The patient fibroblasts are ultimately able to extend some abnormally long cilia that are missing key transition zone components. Defective transition zone formation likely disproportionately affects the long-living ciliary outer segment of photoreceptors resulting in retinal dystrophy. CEP162 is expressed in human retina, and we show that wild-type CEP162, but not truncated CEP162-E646R ${ }^{\star} 5$, specifically localizes to the distal end of centrioles of mouse photoreceptor cilia. Together, our genetic, cell-based, and in vivo modeling establish that CEP162 deficiency causes retinal ciliopathy in humans.

Keywords: CEP162, photoreceptor, primary cilium, ciliogenesis, retinal ciliopathy. 


\section{SIGNIFICANCE STATEMENT}

Ciliopathies often comprise retinal degeneration since the photoreceptor's outer segment is an adapted primary cilium. CEP162 is a basal body protein required for proper transition zone assembly during ciliogenesis and has so far not been implicated in human disease. Using genetic, cell-based, and in vivo studies, we show that a biallelic mutation of CEP162 causes late-onset human retinal degeneration. This mutation specifically hinders CEP162 function at the cilia, leading to impaired transition zone assembly and delayed formation of dysmorphic cilia. Studies in mouse photoreceptors support that absence of CEP162 could lead to defective outer segments. The discovery of novel ciliopathy genes, such as CEP162, advances our insight into cell-specific functions of the primary cilium. 


\section{INTRODUCTION}

Retinitis pigmentosa (RP) is a leading cause of visual impairment and the most prevalent inherited retinal disease (IRD), with a worldwide prevalence of approximately $1: 4,000$ (1). It is characterized by primary and predominant degeneration of rod photoreceptors, followed by loss of cone photoreceptors. RP starts with night blindness, followed by progressive concentric loss of visual field and acuity. There is extensive genetic heterogeneity; about 100 genes are currently known to cause nonsyndromic RP (1). Comprehensive targeted analysis of the coding regions of all known IRD genes, either via targeted enrichment and next-generation ('gene panels') or whole-exome sequencing (WES) has become a first-tier diagnostic approach, with solve rates of up to $70 \%$ for IRDs in general and for RP specifically in different populations (2-6). In about $30 \%$ of RP patients, the genetic cause remains unknown, likely due to mutations in many, possibly hundreds, of unidentified RP genes, each accounting for only a small fraction of patients. Given the progress of therapeutic approaches for IRD (7), the identification of the unknown genes and an early diagnosis represents an important challenge.

The genes implicated in RP pathogenesis encode proteins of various functions such as visual cycle, phototransduction, splicing, nucleotide synthesis, transcription, phagocytosis, or channels for ion and small molecule transport. Moreover, about onethird of the genes knowingly associated with an IRD encode for ciliary photoreceptor proteins (8). Primary cilia are antenna-like protrusions of the cell surface that transduce extracellular stimuli to the cell. Dysfunctions of this organelle are associated with a wide range of human disorders collectively termed ciliopathies. Ciliopathies may involve several organs including the kidneys, heart, liver, brain, pancreas, bones and retina. Isolated or syndromic IRD account for more than half of human ciliopathies (9- 
11). These retinal ciliopathies result from defective formation, function and/or maintenance of the light-sensitive photoreceptor outer segment, a modified primary cilium. This leads to photoreceptor degeneration, progressive visual impairment and can ultimately result in blindness.

CEP162 (MIM 610201) is a microtubule-associated protein of the centriole distal ends that promotes assembly of the transition zone, a proximal region of the cilium that features electron dense Y-links connecting the axonemal microtubules to the membrane, by mediating association of transition zone components with microtubules. Loss of CEP162 has been shown to arrest ciliogenesis at the stage of transition zone assembly and causes a ciliopathy phenotype in zebrafish (12). Despite its important function for cilia formation, no pathogenic CEP162 variants have been reported in human disease, and its role for retinal integrity and function has been unknown. In this study, we show that homozygosity for a rare deleterious frameshift variant in CEP162 causes late-onset RP in two distinct Moroccan families, likely representing a founder allele. We observed that the truncated CEP162 retains its localization to the mitotic spindle, but not at the basal body. Absence of CEP162 from the primary cilium results in late formation of dysmorphic cilia that lack transition zone components. Localization in mouse photoreceptors further support that the truncated CEP162 is lost from the basal body and cause this novel retinal ciliopathy. 


\section{RESULTS}

Clinical study. Patient 1 . He reported night blindness that heavily impaired him in dark environment and presumably had preexisted for years. Visual complaints started at the age of 56 years, when the patient noticed loss of visual acuity. It was self-reported that ophthalmological investigations had revealed some abnormalities then, and through more detailed assessment at the age of 60 years, the diagnosis of RP was made. There is now noticeable photophobia. At the age of 67 years, refraction revealed hyperopia and astigmatism in both eyes with +6.25 Sphere (sph), -1.25 Cylinder (cyl) $/ 71^{\circ}$ in the right eye and $+2.75 \mathrm{sph},-1.00 \mathrm{cyl} / 58^{\circ}$ in the left eye. Color fundus photography, blue light autofluorescence and spectral domain optical coherence tomography (OCT) were compatible with RP (pale optic disc, narrow vessels, bone spicule pigmentation; thinning of the outer nuclear layer; Figure 1A-F). Best corrected visual acuity was $20 / 500$ for both eyes (Snellen chart), and visual field testing revealed constricted visual fields. Intraocular pressure was normal under brinzolamide treatment, and anterior segments were unremarkable. There was synchysis scintillans in the vitreous of the right eye. Patient 2. The patient reported visual complaints since the $5^{\text {th }}$ decade, with loss of visual acuity. At the age of 69 , the diagnosis of RP was made. Best corrected visual acuity was light perception (OD) and 4/10 (OS) and visual field testing revealed constricted visual fields $<10^{\circ}$. Color fundus photography showed a pale optic disk, narrow vessels with sheathing and limited bone spicule pigmentation. Fluorescein angiography displayed strong atrophy of the outer retina and few intraretinal pigment migrations. OCT showed absence of the outer retina, except in the fovea. On blue light autofluorescence a Bull's eye aspect was observed. A pattern electroretinogram (ERG) showed an absent response (OD) and reduced macular function (OS), respectively (Figure 1G-N). A full-field ERG could not be conducted. 
Patient 2 was followed at the diabetes clinic for 30 years; no islet cell autoantibodies were detected. There was a low normal production of C-peptide $(0.20 \mathrm{nmol} / \mathrm{L}$. Ref: 0.29-0,99 $\mathrm{nmol} / \mathrm{L})$. He received medication for chronic renal insufficiency due to diabetic nephropathy. Abdominal ultrasound and computed tomography (CT) showed no morphological kidney abnormalities (Supplemental Figure 1), but a lipomatous aspect of the pancreas. Patient 2 suffered from a coronaropathy (main stem stenosis: $50-59 \%$ ), but no surgery was conducted due to a high operative risk. The patient died at the age of 74 .

\section{Genetic study: Homozygous CEP162 frameshift variant in RP patients from two}

unrelated families. Patient 1 and Patient 2 were both born to consanguineous parents in two distinct, not knowingly related, Moroccan families (Figure 2A). Patient 1. The grandparents of Patient 1, a 68-years old male, are $2^{\text {nd }}$ degree cousins. No pathogenic variant was identified in Patient 1 by targeted next-generation sequencing (NGS) of 204 known IRD genes. Subsequent whole-exome sequencing (WES) generated 16.3 $\mathrm{Gb}$ of sequence, covering $97.9 \%$ of the target sequence more than 30x. Among 701 rare variants, 41 were homozygous, and these were further narrowed to 25 by applying a MAF of $<1 \%$, excluding artifacts and filtering with regions of homozygosity $(\mathrm{ROH}$, identified by successive homozygous SNVs across genes). Twenty-four variants were disqualified as cause for RP due to one or several of these reasons: a) The gene was associated with an unrelated disease, b) homo- or hemizygous individuals were annotated in gnomAD, c) the variant was predicted as neutral/benign. One variant remained, a 1-base pair insertion in exon 15 of CEP162, NM_014895.3:c.1935dupA, causing a frameshift and premature nonsense codon (p.(Glu646Argfs $\left.{ }^{\star} 5\right)$, for brevity subsequently designated as $\mathrm{E} 646 \mathrm{R}^{\star} 5$; Figure $\left.2 \mathrm{~B}, \mathrm{D}\right)$. In the other annotated CEP162 isoform, the insertion corresponds to NM_001286206.1:c.1707dup, p.(Glu570Argfs*5). Patient 2. He had an older affected sister, living in North Morocco, who was not 
available for testing. WES in the proband generated 40.6 million reads, with $99.0 \%$ of reads mapping to the target sequences, providing an average coverage of more than 30x. Assessment of 275 RetNet genes did not reveal any (likely) pathogenic variants. An exome-wide analysis revealed 1,298 rare variants (MAF <1\%), 190 of which were homozygous. These were further reduced to 58, filtering with $\mathrm{ROH}$ (Figure $2 \mathrm{C}$, Supplemental Table 1) and ultimately to the CEP162 c.1935dupA variant, based on the aforementioned criteria. Because CEP162 was shown to bind to microtubules during mitosis (13), we analyzed fibroblasts from Patient 1 for chromosome missegregation. No aneuploidy was detected in 30 metaphases from cultured fibroblasts (data not shown).

Patient 1 \& 2. Segregation analysis in both families was in keeping with a pathogenic nature of the truncating CEP162 variant (Figure 2A-B; Supplemental Figure 2) which was absent from gnomAD (v.2.1.1). No homozygous CEP162 LoF variants were found in gnomAD, indicating a recessive gene tolerating heterozygous, but not biallelic deleterious variants. The variant was neither found in 70 RP patients from North Africa (43 of which from Morocco) by targeted testing, nor in WES data from 1,184 IRD cases (Ghent University Hospital). From the ROHs present in Patient 1 and 2, the largest is located on chromosome 6 (Figure 2C; Supplemental Table 1) and includes the CEP162:C.1935dupA variant, further supporting its pathogenicity and putting it forward as a regional founder allele (Figure $2 \mathrm{C}$ ).

\section{C.1935dupA CEP162 mRNA escapes NMD in patient fibroblasts, allowing for} expression of truncated CEP162 protein. To understand how the CEP162 variant c.1935dupA affects gene expression and protein levels, fibroblasts were obtained from Patient 1 and compared to control human dermal fibroblasts (Hdfa). qRT-PCR analysis revealed that CEP162 transcript levels were significantly reduced in patient fibroblasts compared to Hdfa controls (Supplemental Figure 3A). Despite this reduction, CEP162 
mRNA levels significantly increased upon serum starvation, suggesting that the transcriptional regulation of the CEP162 gene remained unaffected (Supplemental Figure 3A). Anisomycin treatment of patient and control fibroblasts was used to determine whether the patient CEP162 transcript undergoes NMD. Increased expression of CEP162 after anisomycin treatment was observed in both patient and control cells (Supplemental Figure 3B), suggesting that a basal level of CEP162 transcript undergoes NMD under physiological conditions.

Because we did not observe increased levels of NMD in patient fibroblasts, we wanted to determine whether the truncated CEP162-E646R ${ }^{\star} 5$ protein was stably expressed. To detect the truncated CEP162 product, we ran control and patient fibroblast lysates on a Western blot and probed with an anti-CEP162 antibody that recognizes the $\mathrm{N}$-terminus prior to the truncation. A band at the predicted size of truncated CEP162 ( 75 kDa) was present in patient lysates compared to a band corresponding to full-length CEP162 ( 160 kDa) in control lysates (Supplemental Figure 3C). In addition, probing with an anti-CEP162 antibody that recognizes the Cterminus produced a full-length CEP162 band in control lysate, but no corresponding or truncated band in the patient lysate (Supplemental Figure 3C). We conclude that despite the lower levels of CEP162 mRNA, the residual transcript does not undergo complete NMD, resulting in stable expression of some residual truncated CEP162E646R ${ }^{\star} 5$ protein in patient cells (Supplemental Figure 3D).

CEP162-E646R ${ }^{\star} 5$ mutant protein is unable to associate with microtubules, centrioles, or CEP290. Human CEP162 comprises 1,403 amino acids with three coiled-coil (CC) stretches in its C-terminus: CC1 (residues 617-906), CC2 (957-1,121) and CC3 $(1,167-1,386)$. CEP162 associates with microtubules through its CC1/CC2 domains and with centrioles through its CC3 domain (12). In the patients, the c.1935dupA mutation results in early truncation of the protein after the first 29 amino 
acids of the CC1 domain (Figure 3A). To determine whether the CEP162-E646R ${ }^{\star} 5$ mutant protein associates with microtubules, we transiently transfected 293T cells with either a FLAG-tagged human CEP162 full-length or E646R ${ }^{\star} 5$ mutant construct with a GFP transfection marker. First, we confirmed expression of full-length FLAG-CEP162 band at $\sim 165 \mathrm{kDa}$ and truncated FLAG-CEP162-E646R ${ }^{\star} 5$ at $\sim 80 \mathrm{kDa}$ when probed with either an anti-CEP162 N-terminal or anti-FLAG antibody (Figure 3B, Supplemental Figure 4A). Immunofluorescence analysis showed full-length FLAG-CEP162 decorating microtubules in cycling 293T cells, as previously described (Figure 3C) (12). However, mutant FLAG-CEP162-E646R5 clustered into puncta throughout GFPpositive cells, suggesting that microtubule association was disrupted in the mutant CEP162-E646R ${ }^{\star} 5$ protein (Figure 3C). To determine whether this truncated mutant protein associates with the centrioles, we co-transfected IMCD3 cells with the FLAGtagged CEP162 constructs as well as a ciliary marker, Htr6-GFP, and serum-starved for 24 hours to induce ciliation (Figure 3D). Immunohistochemical analysis from Htr6GFP positive cilia shows full-length FLAG-CEP162 co-localizing with $y$-tubulin at the basal bodies. In addition, co-staining with a proximal-end centriole protein, CEP250, showed proper localization of FLAG-CEP162 to the distal end of the centrioles. In contrast, the mutant FLAG-CEP162-E646R ${ }^{\star} 5$ was not detected at the $\mathrm{y}$-tubulin-labeled basal bodies or CEP250 labeled proximal-end centrioles (Figure 3D).

It was previously shown that CEP162 interacts with CEP290 through its CC1/CC2 domain (12). To determine whether the mutant CEP162-E646R ${ }^{\star} 5$ protein interacts with endogenous CEP290, we performed FLAG immunoprecipitations from our transfected 293T cells expressing either full length or mutant CEP162. We found that FLAG-CEP162-E646R5 was unable to pull down CEP290; however, CEP290 localization at the centrioles was normal in the patient fibroblasts (Figure 3E, F). In line with this result, no difference in CEP290 protein levels was observed in patient 
fibroblasts compared to controls under serum or serum-free conditions (Supplemental Figure 5).

Patient fibroblasts have delayed ciliation. CEP162 is present at the centrioles throughout the cell cycle, and its microtubule-binding activity (CC1/CC2 domains) is believed to direct its localization to the mitotic spindle during cell division (12). We found that patient fibroblasts expressing the CEP162-E646R ${ }^{\star} 5$ truncated protein had a normal complement of chromosomes and growth rates comparable to control fibroblasts. Interestingly, although truncated CEP162-E646R ${ }^{\star} 5$ lacks most of the CC1/CC2 domains, we found that CEP162 staining decorated the microtubule spindles of dividing cells in the patient fibroblasts similar to controls (Figure 4A). The absence of aneuploidy in the patient fibroblasts suggests that CEP162 is dispensable for intact mitosis.

It was previously shown that siRNA CEP162 knockdown results in decreased ciliation in RPE1 cells (12). CEP162 co-localizes with y-tubulin-positive centrioles at the base of Arl13b-labelled cilia in control fibroblasts. In patient fibroblasts, although Arl13b staining accumulated at the centrioles, CEP162 was not detected, and no cilium was formed (Figure 4B). Acetylated tubulin staining was observed within these abnormal Arl13b accumulations but did not extend along an axoneme as seen in control fibroblasts (Figure 4B). This suggests that ciliogenesis is halted prior to axoneme extension in the patient fibroblasts. To determine whether the patient fibroblasts could eventually produce cilia, we serum-starved for up to 72 hours. While control fibroblasts produced cilia within 24 hours, very few cilia in patient fibroblasts were observed before 72 hours serum withdrawal. However, by 72 hours the number of cilia produced in the patient fibroblasts was not significantly different from controls (Figure 4C). Ciliary length was measured for every cilium imaged at the three timepoints (Figure 4D). The cilia in patient fibroblasts at 72 hours were significantly 
longer than in controls ( $p>0.0001)$. Representative images of cilia from control and patient fibroblasts stained with Arl13b and $\mathrm{y}$-tubulin are shown in Figure 4E. In addition, staining for polyglutamylated tubulin (B3), a tubulin modification present in the axoneme, was not observed at Arl13b-positive centrioles of patient fibroblasts but was present in the extra-long cilium of the patient fibroblasts (Supplemental Figure 4B). Together, our data suggest that in patient fibroblasts, ciliogenesis is paused before axoneme extension; however, these cells can ultimately overcome the loss of CEP162 at the centrioles and produce a cilium, although abnormal in length.

We used correlative light and scanning electron microscopy (CLSEM) to examine the structure of the arrested cilia in the patient cells and to compare them to normal cilia produced in controls after 48 hours of serum withdrawal (Figure 4F). While we observed Arl13b-positive primary cilia of normal length in control fibroblasts, the patient fibroblasts only produced Arl13b-positive blebs on the cells' surface. This suggests that although the axoneme has yet to elongate in the patient fibroblasts, the ciliary membrane has fused with the plasma membrane, resulting in a bulbous bleb on the cell's surface. Accumulation of ciliary membrane before axoneme elongation could affect the final length of the cilium in the patient fibroblasts.

Persistence of $\mathrm{CP} 110$ at the mother centriole causes arrest of primary ciliogenesis in patient fibroblasts. Ciliogenesis is a highly coordinated multi-step process (14). To determine the stage of ciliogenesis when it is arrested in fibroblasts of our patient, we analyzed the localization of molecular components involved in cilia formation. First, maturation of the basal body appears to be normal as proteins involved in ciliary vesicle fusion (EHD1), ciliary membrane growth (Rab8a) and distal appendages formation (CEP164) were normal in the patient fibroblasts (Figure 5A). Since the patient fibroblasts accumulated Arl13b, a protein recruited to the ciliary vesicle along with the IFT machinery, we next stained for IFT88, a component of 
complex B present in the pericentriolar region and in IFT trains within the primary cilium. Figure 5A shows normal localization of IFT88 in patient fibroblasts, suggesting that steps leading to ciliary vesicle formation proceed normally and that the IFT machinery is unaffected by the loss of CEP162 in the cilium.

Following ciliary vesicle formation, CP110, a distal-end centriole protein that prevents microtubule nucleation, must be removed from the mother centriole and degraded to allow for proper axoneme elongation (15). This key regulatory step was previously reported to be unaffected by loss of CEP162 in RPE1 cells (12). In the patient fibroblasts, CP110 staining revealed persistence of $\mathrm{CP} 110$ on the mother centriole (Figure 5B, C). In addition, Western blot analysis for CP110 indicates that while levels normally decrease $50 \%$ upon serum starvation in controls, levels of CP110 remained unchanged in the patient fibroblasts 24 hours after serum withdrawal (Figure 5D; Supplemental Figure 5). CP110 removal is controlled by CEP164-mediated recruitment of the serine/threonine protein kinase, TTBK2, to the distal appendages $(16,17)$. TTBK2 phosphorylates multiple targets, such as CEP83 and MPP9 $(15,18)$, that are required for removal of CP110 and initiation of ciliogenesis. We found both TTBK2 and its MPP9 substrate were normally localized at the basal body of the patient fibroblasts (Figure 5E). Together, these data suggest that although the molecules needed to remove CP110 from the mother centriole are present, this process is delayed in the patient fibroblasts.

It was previously reported that overexpression of C-terminally truncated CEP162, maintaining microtubule association but unable to localize to centrioles, resulted in a higher fraction of RPE1 cells producing cilia that were abnormally long. In these experiments, the truncated CEP162 was found at the axoneme tip of elongated cilia where it ectopically recruited transition zone components (12). We found that E646R ${ }^{\star} 5$-truncated CEP162 protein maintains microtubule localization during mitosis 
but is not bound to centrioles, suggesting it may behave in a similar manner as reported by Wang et al. (12). To determine if the abnormally long cilia resulted from mislocalization of transition zone components to the axoneme tip, we stained control and patient fibroblasts for four transition zone proteins: TMEM67, NPHP1, RPGRIP1L, and TCTN1 (Figure 5F). In control fibroblasts, these proteins showed a normal position distal to the mother centriole. In patient fibroblasts, the localization of TCTN1 was also normal; however, TMEM67, NPHP1 and RPGRIP1L were not properly assembled at the ciliary base. These results are similar to what was shown in cells lacking CEP162 (12). Additionally, we did not find mislocalization of any of these transition zone components at the tip of the long patient cilia (Figure 5F). These data indicate that the truncation of CEP162 results in loss of its ciliary function in patient cells.

To confirm that CEP162-E646R ${ }^{\star} 5$ has no ciliary function, we performed shRNA knockdown of CEP162 in IMCD3 cells, followed by rescue with either FLAG-tagged full-length CEP162 or CEP162-E646R ${ }^{\star} 5$. To identify transfected cells, we generated a single plasmid to co-express shRNA and soluble mCherry. In addition, we cotransfected these plasmids with an Htr6-GFP plasmid to label the cilia. Figure 5G shows representative images of Htr6-GFP cilia of mCherry-positive cells when expressing a control or CEP162 shRNA. CEP162 knockdown resulted in reduced ciliation compared to control shRNA (Figure 5H). Expression of the FLAG-tagged fulllength CEP162 rescued the loss of cilia due to CEP162 knockdown; however, FLAGCEP162-E646R ${ }^{\star} 5$ was unable to rescue (Figure 5G, H). Together, our results support that even though the CEP162-E646R ${ }^{\star} 5$ protein is it expressed; it does not retain any function at the cilium.

\section{CEP162 is expressed in human retina and localizes to the basal body in mouse}

photoreceptors. We examined CEP162 expression in single-cell (sc) transcriptional data of human neural retina (19). This revealed high expression in all retinal cell types, 
especially in ganglion cells (Supplemental Figure 6A). Immunostaining for CEP162 on human retinal sections shows ubiquitous expression throughout the human retina (Supplemental Figure 6B-F), similarly to expression previously determined in chick retina (20). CEP162 is a centriolar protein; to determine its precise subcellular localization in photoreceptor cells, we used Cetn2-GFP transgenic mice that have fluorescently labelled centrioles in cells of every organ (21). In photoreceptors, mother and daughter centrioles form two adjacent GFP dots, and the connecting cilium emerges as a GFP streak from the mother centriole. CEP162 staining is localized to the distal end of both mother and daughter centrioles at the photoreceptor basal body (Figure 6A). Airyscan images were acquired to determine the precise localization of CEP162 in relation to several ciliary markers: Acetylated tubulin for the axoneme, CEP290 for the connecting cilium, CEP164 for the distal appendages, and CP110 for the daughter centriole (Figure 6B). These images show that CEP162 decorates the distal ends of each centriole of the basal body at the base of the photoreceptor outer segment.

To determine whether the truncated CEP162-E646R* 5 mutant protein is able to localize to the basal body in photoreceptors, we employed an in vivo electroporation approach (22) to express FLAG-tagged full-length and E646R*5-mutant CEP162 constructs in mouse rod photoreceptors. We co-expressed mCherry-tagged rhodopsin to label the outer segment in transfected rods and stained retinas with anti-Centrin1 antibodies to label the connecting cilium in all photoreceptors. Figure 6C shows FLAGCEP162 staining at the basal body directly below the connecting cilium only in rods with Rho-mCherry-labelled outer segments. In contrast, FLAG-CEP162-E646R ${ }^{\star 5}$ mutant staining does not localize to the basal body of Rho-mCherry-labelled outer segments (Figure 6D). In line with the expression in IMCD3 cells, this suggests that 
the truncated CEP162-E646R ${ }^{\star} 5$-mutant protein is unable to properly localize to the centrioles in rod photoreceptors, supporting the retinal ciliopathy phenotype in humans. 


\section{DISCUSSION}

CEP162 was originally cloned from quail retinal neurons and hence initially designated quail neuroretina 1, QN1. Expression of QN1 was shown to be neuronspecific and correlated with the withdrawal from cell cycle, so QN1 was postulated to play a role in neuronal quiescence (20). QN1 is orthologous to human KIAA1009/CEP162 (23). CEP162 was shown to bind to microtubules during mitosis and specifically localizes at the distal ends of centrioles in post-mitotic cells. Detailed functional investigation showed that complete loss of CEP162 or expression of truncated CEP162 proteins did not affect mitosis or centriole duplication, compatible with the lack of aneuploidy and with normal growth observed in patient-derived fibroblast cultures. Instead, CEP162 was shown to play a functional role in ciliogenesis by promoting transition zone assembly (12). Here, we identified a homozygous frameshift variant in RP patients from two distinct families and provide strong functional evidence supporting the categorization of CEP162 as a novel retinal ciliopathy gene.

CEP162 is expressed in human retina and localizes to the centrioles forming the photoreceptor outer segment in mouse retina. In patient-derived fibroblasts, low levels of CEP162 mRNA were preserved and sufficient to produce an - albeit heavily truncated - CEP162 protein. Knockdown of Cep162 impairs transition zone assembly and thus ciliogenesis in cell culture, while morpholino knockdowns in zebrafish resulted in a ciliopathy phenotype (12). In our study, we show that in patient fibroblasts the residual truncated CEP162 protein was present at the mitotic spindles, but not at the ciliary basal body. While this suggests that truncated CEP162 could maintain some function at the mitotic spindle, we show through rescue experiments that the truncated CEP162 is unable to restore the loss-of-function phenotype at the cilium. We further show that truncated CEP162-E646R*5 is unable to bind CEP290. 
We found that cilia were unable to extend an axoneme because CP110 persisted at the mother centriole. CP110 caps the distal end of centrioles, and its removal and degradation are prerequisites for ciliation. Other aspects of early ciliogenesis, like the acquisition of distal appendages and fusion of the ciliary vesicle to the plasma membrane, were unaffected in patient's fibroblasts. Interestingly, a dramatic increase in ciliation after extended serum starvation suggests that in least some patient cells CP110 was ultimately removed, and abnormally long cilia extend.

It is interesting to speculate that perhaps the RP-causing defect in the patient (with residual CEP162 function) is not in only impaired ciliogenesis per se, as some cilia can still be formed, but also lack of recruitment of key transition zone proteins, such as NPHP1, to the primary cilium. This could result in structural defects that are particularly detrimental to the photoreceptor cilium, impairing photoreceptor maintenance rather than formation - clinically manifesting as late-onset RP and not as congenital retinal disease (as in CEP290-associated Leber's congenital amaurosis $(24))$.

In summary, we have established specific CEP162 deficiency at the cilium as a novel cause of human retinal ciliopathy by genetic, cell-based, and in vivo modeling findings. Identification of ciliopathy genes advances our insight into the primary cilium, an essential organelle involved in many human disorders, which is a prerequisite for the development of therapeutic strategies for this large disease group. 


\section{MATERIALS AND METHODS}

Patients. All individuals involved gave their informed consent prior to inclusion in this study. All investigations were conducted according to the Declaration of Helsinki, and the study was approved by the Institutional Review Board of the Ethics Committee of the University Hospital of Cologne, Ghent (EC UZG 2017/1540) and Brussels (A420701El13L).

Clinical assessment. Affected individuals were subjected to ophthalmologic evaluation. For Patient 1, ophthalmic imaging included slit lamp examination, widefield color fundus photography and blue light autofluorescence (CLARUS 500; Carl Zeiss Meditec Inc., Dublin, CA), as well as spectral domain optical coherence tomography (Spectralis SDOCT, Heidelberg Engineering, Germany; PLEX Elite 9000; Carl Zeiss Meditec Inc., Dublin, CA). Patient 2 was investigated accordingly. Patients 1 and 2 underwent abdominal ultrasound to exclude cysts of the kidneys and liver fibrosis. Patient 2 also underwent abdominal CT.

Genetic studies in Families 1 and 2. For the index patients of both families, Patients 1 and 2, genomic DNA (gDNA) was extracted from leukocytes, and pooled libraries were paired-end sequenced on an Illumina NextSeq500 platform (Illumina, San Diego, CA). Nucleotides were numbered following HGVS guidelines (http://www.hgvs.org) with nucleotide 'A' of the ATG as ' $c .1$ '. The cut-off for the maximum minor allele frequency (MAF) was 1\%. Variants were checked for their presence in the ClinVar and HGMD databases $(25,26)$ and classified based on the ACMG and ACGS guidelines with adaptations (27-30). Patient 1. Targeted NGS (enrichment with IDT xGen® Inherited Diseases Panel v1.0; IDT Integrated Technologies, Coralville, IA), WES (IDT xGen® Exome Research Panel v1.0) and variant assessment were conducted as reported previously (31). After targeted NGS, variant analysis was performed for 204 
genes knowingly associated with IRD, including RPGR with ORF15 (SeqNext module of SeqPilot software, JSI Medical Systems). Genes associated with the Human Phenotype Ontology (HPO) term "Retinal dystrophy" (HP:0000556) were searched for pathogenic homozygous or potentially compound-heterozygous variants (varSEAK Pilot 2.0.2, JSI Medical Systems). After WES, the CCG pipeline (32) and interface (Varbank 2.0) were used for data analysis as described $(33,34)$. ExAC and gnomAD databases (35) (as of 02/2021) were searched for candidate variants identified by targeted NGS and WES in homozygous state. Patient 2. WES was performed using the SureSelect XT Human All Exon V6 kits (Agilent, Santa Clara, CA, USA) for exome enrichment. The CLC Genomics Workbench version 7.0.5 (CLCBio, Aarhus, Denmark) was used for read mapping against the human genome reference sequence (National Center for Biotechnology Information, GRCh37/hg19), removal of duplicate reads after mapping, coverage analysis, and quality-based variant calling, followed by further variant annotation using Alamut Batch (Interactive Biosoftware, Rouen, France). Variants were scored heterozygous or homozygous and assessed with our in-house variant filtering and visualization tool. A selection of 275 RetNet genes was assessed respectively (version 4 of the RetNet panel) followed by assessment of homozygous variants in the exome. Copy number variations (CNVs) were assessed using ExomeDepth (v1.1.10) (36). Segregation analysis and screening for the CEP162 variant c. $1935 d u p A$ p. $\left(E 646 R^{*} 5\right)$ in IRD patients. Available members of Families 1 and 2 and 70 RP patients from North Africa, including 43 from Morocco, were tested for the CEP162 variant c.1935dupA p.(E646R $\left.{ }^{\star} 5\right)$ by Sanger sequencing (primer sequences: Supplemental Table 1). Pseudonymized WES data from 1,184 IRD cases were also analyzed for presence of the variant (Ghent University Hospital).

Run of homozygosity $(\mathrm{ROH})$ detection and haplotype analysis. Patient 1. $\mathrm{ROH}$ were identified using HomozygosityMapper applying default settings (37). $\mathrm{ROH}<3 \mathrm{~kb}$ 
adjacent to each other were merged manually which reduced the number of $\mathrm{ROH}$ from 71 to 67 and increased the block size of the largest $\mathrm{ROH}$ from $15.8 \mathrm{Mb}$ to $16.0 \mathrm{Mb}$. Patient 2. $\mathrm{ROH}$ mapping was initially performed using the genome-wide singlenucleotide polymorphism (SNP) chip analysis using the HumanCytoSNP-12 BeadChip platform (Illumina, San Diego, CA). Homozygous regions (>1 Mb) were identified using PLINK software (38) integrated in ViVar (39). Resulting homozygous regions were ranked according to their length and number of consecutive homozygous SNPs, as described. Patient 1 and 2. ROHs were determined with the AutoMap tool (40) using VCF files from both patients (hg38), with default AutoMap settings. A first analysis determined the individual $\mathrm{ROH}$ per patient, followed by a second analysis where shared $\mathrm{ROH}$ (coordinate-wise) were assessed. AutoMap output consists of a graphical representation of $\mathrm{ROH}$ (Figure $2 \mathrm{C}$ ) as well as a text file reporting the numerical values of the $\mathrm{ROH}$ positions with their size, number of variants, and percentage of homozygosity (Supplemental Table 1). To assess the identity of the shared $\mathrm{ROH}$ on chromosome 6 and of a common haplotype containing the CEP162 variant, all variants on chromosome 6 detected by WES were inspected, irrespective of their zygosity (Figure 2C).

CEP162 expression in human retinal single-cell transcriptional datasets. Singlecell (sc) transcriptional datasets generated from adult human peripheral and foveal retina were mined for evaluating CEP162 expression at the sc level (Supplemental Figure 6A). Expression matrices derived from pooling 3 donor retinas were retrieved and processed as described (19), resulting in 19,768 cells after quality control preprocessing. Following dimensionality reduction, embedding and clustering, CEP162 expression was evaluated at sc-level using markers associated with major retina cell populations. All analyses were performed with SCANPY (v1.4.6) (41). 
Fibroblast culture from skin biopsy. Fibroblasts were isolated from a dermal punch biopsy of the upper arm of Patient 1 as previously described (42), following Institutional Review Board approval and patient's informed consent. In short, the skin biopsy was cut in 18-24 equally sized pieces containing all skin layers. The pieces were plated on a six well plate that was previously coated with $0,1 \%$ Gelatin. Fresh medium was added every day. Fibroblasts started to migrate out of the skin biopsies after 7-10 days and were split on two $75 \mathrm{~cm}^{2}$ flasks after 3-4 weeks. When reaching $90 \%$ confluency, the two flasks were split on three $175 \mathrm{~cm}^{2}$ flasks. From here, fibroblasts could be expanded as needed or were frozen in liquid nitrogen for long-term storage and later experiments. After isolation, fibroblasts were routinely cultured in IMDM/Glutamax containing 15\% fetal bovine serum (FBS) and 1\% penicillin/streptomycin (all Invitrogen, Waltham, MA). Normal Primary Human Dermal Fibroblasts (HDFa, ATCC \#PCS-201-012; Gaithersburg, MD) were purchased and used for controls.

Karyotyping. Karyotyping was carried out for 30 metaphases from cultured Patient 1 fibroblasts, following standard procedures.

Assessment of nonsense-mediated decay (NMD). To inhibit NMD, cultured fibroblasts $(80-90 \%$ confluence) of the patient and a proband were incubated with anisomycin $(1 \mu \mathrm{g} / \mathrm{ml})$ for $6 \mathrm{~h}$. RNA isolation and reverse transcription were performed as described previously (43). The expression change of CEP162 and an NMD control transcript after anisomycin treatment was analyzed by digital droplet PCR (ddPCR; QX200 Droplet Digital PCR System, Bio-Rad Laboratories; Hercules, CA) (for primers, see Supplemental Table 2).

Animals. Mice were handled following the protocols approved by the Institutional Animal Care and Use Committees at the University of Michigan (registry number A3114-01). Albino Cetn2-GFP transgenic mice used in immunofluorescence experiments were obtained from Jackson Labs (Strain \# 027967; Bar Harbor, ME). The 
$P d e 6 b^{R d 1}$ mutation was removed by backcrossing to a BALB/cJ albino mouse from Jackson Labs. Albino CD-1 wild type mice used in electroporation experiments were obtained from Charles River (Strain \# 022; Mattawan, MI). All mice were housed under a 12/12-hour light cycle. The experimenters were not blinded to genotype.

In vivo electroporation: Retinal transfection of neonatal mice by the in vivo electroporation technique (22) was conducted with previously described modifications $(44,45)$ to express exogenous constructs in mouse rods. DNA constructs were electroporated into the retinas of wild-type CD-1 neonatal mice. Following anesthetization of neonatal mice (P0-P2) on ice, the eyelid and sclera were punctured at the periphery of the eye using a blunt-end 30-gauge needle. It was advanced through the puncture wound until reaching the subretinal space, and $0.3-0.5 \mu \mathrm{l}$ of concentrated plasmid DNA $(2 \mu \mathrm{g} / \mu \mathrm{l}$ of the respective construct and $1 \mu \mathrm{g} / \mu \mathrm{l}$ rhodopsinmCherry to visualize transfected cells) was deposited. A tweezer-type electrode (BTX; Holliston, MA) was placed over the mouse's head with the positive electrode overlying the injected eye. Five $100 \mathrm{~V}$ pulses of 50 milliseconds duration were applied using an ECM830 square pulse generator (BTX). Neonates were returned to their mother and allowed to develop until postnatal day 21 when mice were sacrificed by $\mathrm{CO}_{2}$ inhalation, followed by decapitation and retinal tissue collection for analysis.

Primary antibodies. pAb anti-CEP162 and pAb anti-NPHP1 antibodies were generously provided by Brian Tsou, Sloan Kettering Institute (12), and by Greg Pazour, University of Massachusetts Medical School (46), respectively. A complete list of commercial antibodies and dilutions used can be found in Supplemental Table 3.

Quantitative RT-PCR. Human control and patient cells were collected, RNA extracted using NucleoSpin RNA Kit (TaKaRa; Mountain View, CA) and adjusted to $50 \mathrm{ng} / \mathrm{\mu l}$. cDNA was then synthesized from total RNA using iScript Advanced cDNA Synthesis Kit (Bio-Rad). Real time PCR was performed in a $10 \mu \mathrm{l}$ reaction volume with $0.5 \mu \mathrm{l}$ of 
cDNA using the iTaq Universal SYBR Green Supermix (Bio-Rad). Thermal cycling and SYBR detection were performed on a CFX96 Real Time System (Bio-Rad). A complete list of these primers can be found in Supplemental Table 2. All statistical analysis was performed as unpaired t-test using GraphPad Prism 9 (GraphPad Software, LLC).

DNA constructs. DNA constructs were generated using standard PCR-based subcloning methods. Homo sapiens centrosomal protein 162 (CEP162), transcript variant 1, mRNA (Accession: NM_014895.3) was obtained from GeneCoepia (HOC20483; Rockville, MD). An N-terminal FLAG-tag was added by overlap extension PCR. All DNA constructs were cloned between a 5' Agel and a 3' Notl site, using standard T4 DNA ligation methods, and underwent confirmatory sequencing. For mouse in vivo electroporation, the pRho plasmid driving gene expression under the 2.2 kb bovine rhodopsin promoter was used (Addgene, plasmid \# 11156; Watertown, MA). For mammalian cell culture, the pEGFP-N1 vector was used (Clontech, PT3027-5; Mountain View, CA). All forward and reverse primers were designed to introduce $5^{\prime}$ Agel and 3' Notl sites, respectively. Point mutations were generated using the QuikChange II XL kit (Stratagene, La Jolla, CA). The rhodopsin C-terminally tagged with mCherry was previously used (47). A complete list of primers can be found in Supplemental Table 2.

Plasmid expression in cell culture. AD293T or IMCD3 cells were transfected at 9095\% confluency with DNA constructs mentioned above using Lipofectamine 3000 transfection reagent (Invitrogen, L3000001). The next day cells were incubated in complete media or serum-free media for another 24 hours before analysis.

Immunofluorescence. Cetn2-GFP mouse retinal cross-sections: Immunostaining was carried out for connecting cilium proteins as previously described (48). Briefly, posterior eyecups were embedded in OCT compound without fixation and quick-frozen in methylbutane cooled with liquid nitrogen. $10 \mu \mathrm{m}$ thick cross-sections through the 
central retina were cut using a cryostat (Leica Biosystems; Buffalo Grove, IL), collected on pretreated glass slides (Superfrost Plus; Thermo Fisher Scientific, Waltham, MA) and stored at $-80^{\circ} \mathrm{C}$. For immunostaining, sections were blocked in $5 \%$ donkey serum and $0.05 \%$ Triton $\mathrm{X}-100$ in PBS for 15 minutes at $22^{\circ} \mathrm{C}$ before incubating in primary antibody diluted in blocking solution for 1 hour at $22^{\circ} \mathrm{C}$. After primary antibody incubation, the sections were rinsed 3 times in PBS and then fixed for 5 minutes in 1\% paraformaldehyde in PBS. Fixed sections were rinsed 3 times in PBS and then incubated with $10 \mu \mathrm{g} / \mathrm{ml}$ Hoechst 33342 and donkey secondary antibodies conjugated with Alexa Fluor 488, 568, or 647 (Jackson ImmunoResearch; West Grove, PA) in blocking solution for 2 hours at $22^{\circ} \mathrm{C}$. Sections were mounted with Prolong Glass Antifade Mountant (Thermo Fisher Scientific) and cover-slipped (\#1.5, Electron Microscopy Sciences; Hatfield, PA) for imaging.

Electroporated mouse retinal cross-sections: Immunostaining was carried out using a protocol adapted from Robichaux et al. (49). Electroporated retinas with mCherry expression were dissected in $1 \times$ Mouse Ringers, $\mathrm{pH} 7.4$ supplemented with $10 \mathrm{mM} \mathrm{D}$ glucose, $0.5 \mathrm{mM}$ Sodium glutamate, $3 \mathrm{mM}$ Sodium Succinate, $20 \mathrm{mM}$ bicarbonate and $1 \times$ vitamins and osmolarity adjusted to $\sim 313-320 \mathrm{mOsM}$. Unfixed retinas were then blocked in $10 \%$ normal donkey serum $+0.3 \%$ saponin $+1 \times$ cOmplete $^{\mathrm{TM}}$ Protease Inhibitor cocktail (Millipore Sigma; Burlington, MA) diluted in $1 \times$ Supplemented Mouse Ringers for 2 hours at $4^{\circ} \mathrm{C}$. Primary antibodies were added to the blocking buffer and incubated at $4{ }^{\circ} \mathrm{C}$ for 20 to $22 \mathrm{~h}$. For antibody information, see Supplemental Table 3. Retinas were washed and $10 \mu \mathrm{g} / \mathrm{ml}$ Hoechst and donkey secondary antibodies conjugated with Alexa Fluor 488 or 647 were incubated for 2 hours at $4^{\circ} \mathrm{C}$. Retinas were washed and fixed in $4 \%$ paraformaldehyde for 30 minutes at $23^{\circ} \mathrm{C}$. Fixed retinas were then embedded in 4\% agarose (Thermo Fisher Scientific, BP160-500) and 100 $\mu \mathrm{m}$ cross-sections through the transfected retinal patch were collected using a 
vibratome (Leica Biosystems VT1200S), mounted with Prolong Glass and coverslipped.

Human retinal cross-sections: Retinal samples of human donors were obtained from the Eye Bank of the Center of Ophthalmology, University of Cologne, Germany. The research followed the tenets of the Declaration of Helsinki. After dissection of the anterior segment, the remaining tissue included the posterior pole. Remaining vitreous humor was removed to obtain retinal tissue. For immunofluorescence analysis, horizontal retinal cryosections were fixed with $4 \%$ paraformaldehyde and rinsed with PBS. Sections were then rehydrated in PBS and preincubated with $1 \%$ dried milk in PBS and $0.01 \%$ Tween 20 to reduce nonspecific immunoreactivity. Overnight incubation with the primary anti-CEP162 antibody (HPA030172, Sigma-Aldrich, St. Louis, MO) was performed at $4{ }^{\circ} \mathrm{C}$ in PBS containing $2 \%$ BSA, $0.02 \%$ NaN3 and $0.1 \%$ Triton $\mathrm{X}-100$. To estimate the specificity of the primary antibody, control stainings without primary antibody were performed in parallel. After washing in PBS, samples were labeled for $1 \mathrm{~h}$ at room temperature with the secondary anti-rabbit antibody conjugated to Alexa594 (red) (Dianova, Hamburg, Germany). Nuclei counter-staining was performed with $0.1 \mathrm{mg} / \mathrm{ml}$ DAPI (4',6-diamidino-2-phenylindole) in PBS (Molecular Probes, Life Technologies, Frankfurt, Germany) for $10 \mathrm{~min}$ at room temperature. The cryosections were mounted with fluorescent mounting medium (Dako Cytomation, Hamburg, Germany) and viewed with a Zeiss Axio Imager.M2 fluorescence microscope equipped with ApoTome.2 (Carl Zeiss, Jena, Germany). Microscopic pictures were analyzed with ZEN software (Carl Zeiss, Jena, Germany) (Supplemental Figure 6B-E).

Cultured cells: $50-60 \times 10^{3}$ cells were plated onto $13 \mathrm{~mm}$ poly-L-lysine glass coverslips (Corning, 354085; Glendale, AZ) in a 24-well plate and grown overnight at $37^{\circ} \mathrm{C}$ with 
$5 \% \mathrm{CO}_{2}$. To image primary cilia, medium was replaced with serum-free DMEM and cells were then grown in the serum-free medium for either 24,48 or 72 hours. Cells were placed on ice for 10 minutes to depolymerize microtubules before fixation in $1 \%$ paraformaldehyde for 5 minutes followed by 15 minutes in ice-cold methanol. Cells were then washed with $1 \times$ PBS, permeabilized for 5 minutes with $0.1 \%$ SDS in $1 \times$ PBS, washed with $1 \times$ PBS and nonspecific binding was blocked with a 1 hour incubation in $1 \times$ PBS containing $0.1 \%$ Triton-X, 5\% normal donkey serum and $5 \%$ BSA. Coverslips were then incubated overnight at $4^{\circ} \mathrm{C}$ with primary antibody diluted in blocking buffer (for antibody information, see Supplemental Table 3), washed in 1x PBS, and incubated for 1 hours at $22^{\circ} \mathrm{C}$ with $10 \mu \mathrm{g} / \mathrm{ml}$ Hoechst and donkey secondary antibodies conjugated with Alexa Fluor 488, 568, or 647 . Coverslips were washed in $1 \times$ PBS and mounted on slides with Prolong Glass for imaging.

Images were acquired using a Zeiss Observer 7 inverted microscope equipped with a $63 \times$ oil-immersion objective (1.40 NA), LSM 800 confocal scanhead outfitted with an Airyscan superresolution detector controlled by Zen 5.0 software (Carl Zeiss Microscopy; White Plains, NY). Manipulation of images was limited to adjusting the brightness level, image size, rotation and cropping using FIJI (ImageJ, https://imagej.net/Fiji).

CEP162 immunohistochemistry on human retina. Adult human retina was fixed in $10 \%$ neutral buffered formaldehyde and embedded in paraffin. Immunostaining of

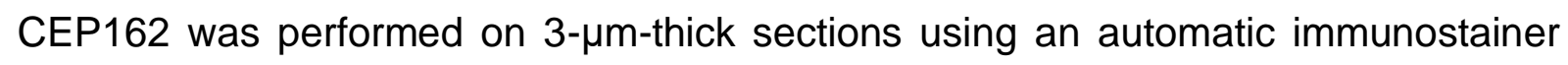
(BenchMark Ultra, Ventana Medical Systems, Tucson, AZ). The rabbit polyclonal antibody anti-CEP162 (HPA030172, Sigma-Aldrich) was used, and visualization was achieved with the OptiView Amplification Kit (Ventana Medical Systems). Heatinduced epitope retrieval was performed using Cell Conditioning 2 (Ventana Medical Systems) (Supplemental Figure 6F). 
Immunoblotting. Cultured cells were grown to $80-85 \%$ confluency in a $15 \mathrm{~cm}$ dish, collected and gently homogenized with a pestle on ice in RIPA buffer $(0.1 \%$ SDS, $0.5 \%$ sodium deoxycholate, 1\% NP-40, $150 \mathrm{mM} \mathrm{NaCl,} 50 \mathrm{mM}$ Tris-HCl, $5 \mathrm{mM}$ EDTA, pH 8.0) with $1 \times$ cOmplete ${ }^{\mathrm{TM}}$ protease inhibitor mixture (Millipore Sigma). Lysates were cleared at $10,000 \mathrm{~g}$ for $20 \mathrm{~min}$ at $4^{\circ} \mathrm{C}$ and total protein concentration was measured using the RC DC Protein Assay kit (Bio-Rad). Lysates were subjected to SDS polyacrylamide gel electrophoresis using a AnykD Criterion TGX protein gel (Bio-Rad) and transferred to Immun-Blot LF PVDF (Bio-Rad). Western blotting was performed by blocking membranes in Intercept blocking buffer (LiCor Bioscience; Omaha, NE) for 1 hour at $22^{\circ} \mathrm{C}$, then incubating in the appropriate primary antibody diluted in $50 \% / 50 \%$ of Intercept / PBST overnight at $4^{\circ} \mathrm{C}$. Blots were then rinsed 3 times with PBST before incubating in the corresponding secondary donkey antibodies conjugated with Alexa Fluor 680 or 800 (LiCor Bioscience) in 50\% / 50\% / 0.02\% of Intercept / PBST / SDS for 2 hours at $4^{\circ} \mathrm{C}$. Bands were visualized and quantified using the Odyssey $\mathrm{CLx}$ infrared imaging system (LiCor Bioscience). Images of the uncropped Western blots can be found in Supplemental Figure 7.

Immunoprecipitation. A $10 \mathrm{~cm}$ plate of AD293T cells was transfected using Polyethyleneimine (PEI, Sigma 408727) at 6:1 ratio PEI:DNA with $10 \mu \mathrm{g}$ of mock, FLAG-CEP162, or FLAG-CEP162-E646R5 plasmid. After 24 hours, cells were lysed in $1 \%$ NP40 in PBS by homogenization with a pestle. Lysates were cleared by centrifugation at $15,000 \mathrm{rpm}$ for $20 \mathrm{~min}$ at $10^{\circ} \mathrm{C}$ and supernatant was incubated on anti-FLAG M2 magnetic beads (Millipore Sigma, M8823) rotating overnight at $4^{\circ} \mathrm{C}$. Immunoprecipitates were eluted with 100 mM 3X FLAG peptide (Millipore Sigma, F4799) in lysis buffer rotating for 1 hour at $4^{\circ} \mathrm{C}$ and then run on an SDS gel and processed for immunoblotting. 
Correlative light and scanning electron microscopy. Control or patient cells were grown in $35 \mathrm{~mm}$ dishes with $14 \mathrm{~mm}$ No. 1.5 gridded glass coverslips (MatTek Life Sciences, P35G-1.5-14-CGRD; Ashland, MA), serum starved for 2 days and fixed for $30 \mathrm{~min}$ at room temperature with $2 \%$ paraformaldehyde, $0.5 \%$ glutaraldehyde, buffered with $0.1 \mathrm{M}$ cacodylate buffer $(\mathrm{CB}), \mathrm{pH} 7.4$ followed by $0.1 \%$ sodium borohydride to quench glutaraldehyde autofluorescence. Immunofluorescence staining was performed as described above using anti-Arl13b antibodies to detect primary cilia. For fluorescence microscopy imaging, cilia were imaged with a 488 laser while DIC images were collected of the corresponding etched gridded coverslip using a Zeiss Observer 7 inverted microscope equipped with a $63 \times$ oil-immersion objective (1.40 NA), LSM 800 confocal scanhead controlled by Zen 5.0 software (Zeiss). Following fluorescent imaging, the cells were fixed overnight at $4^{\circ} \mathrm{C}$ with $2.5 \%$ glutaraldehyde in $0.1 \mathrm{M} \mathrm{CB}$, $\mathrm{pH}$ 7.4. The glutaraldehyde-fixed cells, after washing with $0.1 \mathrm{M} \mathrm{CB}$ three times every 5 minutes, were fixed with $2 \%$ osmium tetroxide (OsO4) in $0.1 \mathrm{M} \mathrm{CB}$ for 5 minutes, washed with $0.1 \mathrm{M} \mathrm{CB}$ three times every 2 minutes, and treated with $2 \%$ tannic acid (TA) in 0.1M CB for 5 minutes at room temperature. This OsO4-TA step was repeated twice to increase more electron conductivity. The OsOs-TA fixed cells were dehydrated through a graded ethanol series to $100 \%$ ethanol. Cells were placed in three changes of hexamethyldisilazane; the final change was allowed to evaporate and fully dry the cells under a fume hood. For scanning electron microscopy, a secondary electron detector of Zeiss EVO 15 LaB6 scanning electron microscope was used to identify the etched gridded coverslips to find the SAME cells imaged for fluorescence and then image at an accelerating voltage of $3 \mathrm{kV}$. SEM processing and imaging was performed at the Microscopy Core by the University of Michigan Medical School. Merged scanning electron microscope and color images were created using the ec-CLEM application in the Icy open-source software. 
Quantification and statistical analysis. All data represent at least three independent experiments. All statistical analyses were performed using Prism9 (GraphPad). The data are presented as a mean +/- SD or SEM as indicated in the figure legends. A Welch's t test was used for all comparisons between control and patient fibroblasts with corresponding $P$ values listed in the legends.

\section{DATA AVAILABILITY}

All unique materials and data that support the findings of this study are readily available from the corresponding authors upon request or from standard commercial sources (as listed in the methods).

\section{ACKNOWLEDGEMENTS}

We are grateful to Woo Jung Cho for assisting the design of CLEM experiments. This work was supported by a NIH P30 grant EY007003 (University of Michigan), NIH T32 grant HD007505 (NN), Matilda E. Ziegler Research Award (JNP), Career Development Award (JNP), an Unrestricted Grant (University of Michigan) from Research to Prevent Blindness, and grants from PRO RETINA Deutschland, from Stiftung Auge (Deutsche Ophthalmologische Gesellschaft; HJB), from Dr. Senckenbergische Stiftung and by Köln Fortune (University Hospital of Cologne; HJB), from Ghent University Special Research Fund (BOF20/GOA/023) (EDB); Ghent University Hospital Innovation Fund NucleUZ (EDB); JED Foundation (EDB); H2020 Marie Sklodowska-Curie Innovative Training Networks (ITN) StarT (grant No. 813490) (EDB). EDB is a senior clinical investigator $(1802220 \mathrm{~N})$ and KVS was a postdoctoral fellow $(12 \mathrm{~W} 7218 \mathrm{~N})$ of the Research Foundation-Flanders (FWO); ADR is Early Starting Researcher of StarT (grant No. 813490). EDB is member of ERN-EYE (Framework Partnership Agreement No 739534-ERN-EYE). 


\section{AUTHOR CONTRIBUTIONS}

NN: Project design, acquisition of data, analysis and interpretation of data, drafting and revising the manuscript.

KVS: Acquisition of data, analysis and interpretation of data, revising the manuscript.

SL: Acquisition of data, analysis and interpretation of data.

MBW: Acquisition of data, analysis and interpretation of data, drafting the manuscript. ADR: Acquisition of data, analysis and interpretation of data, drafting the manuscript. SK: Acquisition of data, analysis and interpretation of data.

MJ: Acquisition of data, analysis and interpretation of data.

JRW: Acquisition of data, analysis and interpretation of data, revising the manuscript.

JW: Acquisition of data, analysis and interpretation of data.

HT: Acquisition of data, revising the manuscript.

NG: Acquisition of data, analysis and interpretation of data.

MVH: Acquisition of data, analysis and interpretation of data.

JW: Acquisition of data, analysis and interpretation of data.

RM: Acquisition of data, analysis and interpretation of data.

SGD: Acquisition of data, analysis and interpretation of data.

JVD: Acquisition of data, analysis and interpretation of data.

$\mathrm{AH}$ : Acquisition of data, analysis and interpretation of data.

HS: Acquisition of data, analysis and interpretation of data.

LM: Acquisition of data, analysis and interpretation of data.

AFR: Acquisition of data, analysis and interpretation of data.

TL: Acquisition of data, analysis and interpretation of data.

KD: Acquisition of data, analysis and interpretation of data.

DR: Acquisition of data, analysis and interpretation of data. 
KMW: Acquisition of data, analysis and interpretation of data.

MVL: Acquisition of data, analysis and interpretation of data.

HR: Acquisition of data, analysis and interpretation of data.

$\mathrm{FH}$ : Acquisition of data, analysis and interpretation of data.

PN: Acquisition of data, analysis and interpretation of data.

HTH: Acquisition of data, analysis and interpretation of data.

UZ: Conception and project design, acquisition of data, analysis and interpretation of data, drafting and revising the manuscript.

JNP: Conception and project design, acquisition of data, analysis and interpretation of data, drafting and revising the manuscript.

EDB: Conception and project design, acquisition of data, analysis and interpretation of data, drafting and revising the manuscript.

HJB: Conception and project design, acquisition of data, analysis and interpretation of data, drafting and revising the manuscript.

\section{COMPETING INTERESTS}

The authors declare no competing interests. 


\section{LEGENDS TO THE TABLES AND FIGURES}

\section{FIGURE 1}

Ophthalmological data of Patients 1 (A-F) and 2 (G-N) showing an RP phenotype.

A Right eye (OD, left column) and B left eye (OS, right column) with pale optic disc, narrow vessels and bone spicule pigmentation on color fundus photography. C, D Granular decreased autofluorescence throughout the posterior pole on blue light autofluorescence. E, F Spectral domain optical coherence tomography (SDOCT) showed cystoid spaces in the inner and outer nuclear layer as well as thinning of the outer nuclear layer with sparing of the central fovea. G Color fundus photography of the left eye, demonstrating a pale optic disc, narrow vessels with pronounced sheathing giving a white (pseudo-thrombotic) aspect, bone spicule pigmentation. $\mathbf{H}$ Fluorescein angiography of the right eye displaying strong atrophy of the outer retina and few intraretinal pigment migrations. Autofluorescence from $\mathbf{I}$ right eye and $\mathbf{J}$ left eye displaying a Bull's eye aspect of the macula. Goldmann visual fields for $\mathbf{K}$ left eye and $\mathbf{L}$ right eye displaying a constriction of $<10^{\circ}$. $\mathbf{M}$ Pattern $E R G$ of left eye displaying reduced macular activity (visual acuity 3/10). For the right eye there were no measurable responses. N OCT showing absence of the outer nuclear layer beyond the macula.

\section{FIGURE 2}

\section{Homozygous CEP162 frameshift mutation causes RP in two unrelated Moroccan}

families. A Pedigrees of the two families reported herein. Individuals whose samples were available were genotyped for the CEP162 pathogenic variant, c.1935dupA (p.(E646R $\left.\left.{ }^{\star} 5\right)\right) . M$, mutation; WT, wild-type. B Electropherograms of an individual with WT sequence (upper panel), Patient 1 (middle; homozygous 1-bp insertion, resulting 
in a frameshift and a premature termination codon after four unrelated residues), and a heterozygous carrier (bottom; all children of the patients). $\mathbf{C ~ R O H}$ on chromosome 6, comprising CEP162, shared by Patient 1 and Patient 2. D Scheme of the CEP162 gene (to scale). Vertical bars indicate exons. The pathogenic variant localizes to exon 15.

\section{FIGURE 3}

\section{Effect of E646R ${ }^{\star} 5$ mutation on CEP162 protein expression and localization. A}

Schematic illustration of human CEP162 protein and its three C-terminal coiled-coil (CC) domains. The predicted truncated CEP162-E646R ${ }^{\star} 5$ mutant protein is depicted below. The corresponding amino acid residues are given for each schematic. B Representative Western blot of 239T cell lysates from untransfected control, transfected FLAG-CEP162 ( 165 kDa, red arrow) and FLAG-CEP162-E646R ${ }^{\star} 5$ ( 80 kDa, red asterisk). Blots were probed for CEP162 to detect expressed protein and $\alpha-$ Tubulin as a loading control. C 293T cells expressing soluble GFP (green) and FLAGCEP162 or FLAG-CEP162-E646R ${ }^{\star} 5$. Transfected cells were identified by GFP (green) fluorescence in the cytoplasm. FLAG (magenta) was co-immunostained with a-Tubulin (aTub, cyan) to label the microtubules. Scale bars, $5 \mu \mathrm{m}$. D Serum starved IMCD3 cells co-expressing Htr6-GFP and FLAG-CEP162 or FLAG-CEP162-E646R5. Transfected cells were identified by GFP (green) fluorescence in the cilium. FLAG (magenta) was co-immunostained with either y-tubulin (cyan, basal bodies) or CEP250 (cyan, proximal-end centriolar protein). E FLAG immunoprecipitation from Mock, FLAGCEP162 or FLAG-CEP162-E646R ${ }^{\star 5}$ transfected 293T cells lysates. Endogenous CEP290 pulls down with full length FLAG-CEP162, but not MOCK or FLAG-CEP162E646R ${ }^{\star} 5$. F CEP290 (magenta) immunostaining in control and patient fibroblasts costained with $y$-tubulin (green). Higher magnification images of the cilium/basal body 
and corresponding staining are shown to the right. Scale bars, $5 \mu \mathrm{m}$ and $2 \mu \mathrm{m}$.

\section{FIGURE 4}

CEP162-E646R ${ }^{\star} 5$ is present at the mitotic spindle in patient fibroblasts, but its basal body absence delays ciliation. A Control and patient fibroblasts co-stained for endogenous CEP162 (magenta) and $\alpha$-Tubulin show CEP162-E646R ${ }^{\star} 5$ protein at the mitotic spindle. Individual stainings are shown to the right. Scale bars $2 \mu \mathrm{m}$. B CEP162 (magenta) immunostaining in control and patient fibroblasts co-stained with Arl13b (green) and either $\gamma$-tubulin or acetylated tubulin (cyan). Scale bars, $5 \mu \mathrm{m}$ and $2 \mu \mathrm{m}$. Higher magnification images and corresponding staining are shown to the right. $\mathbf{C}$ Percent ciliation in control and patient fibroblasts after 24, 48 or 72 hours after serum withdrawal was determined by Arl13b and $\mathrm{y}$-tubulin staining. ${ }^{* *} \mathrm{p}<0.000001,{ }^{* *} \mathrm{p}<0.001$ D Cilia length was measured for each cilium accordingly. ${ }^{* * *} p<0.0001$ Representative cilia from control and patient fibroblasts stained with Arl13b (green) and $\mathrm{y}$-tubulin (cyan). Scale bar, $5 \mu \mathrm{m}$. F Correlative light and scanning electron microscopy were performed in control and patient fibroblasts. Cilia were identified by staining for Arl13b (green). Merged images are shown for a low scanning electron micrograph with magnified micrographs shown on the right. Scale bars, $4 \mu \mathrm{m}$ and 2 $\mu \mathrm{m}$.

\section{FIGURE 5}

CEP162-E646R ${ }^{\star} 5$ does not function at the cilia, yet patient fibroblasts produce dysmorphic cilia at slow rate due to CP110 persistence at mother centriole. A Immunostaining of early ciliogenesis markers in control and patient fibroblasts: EDH1, Rab8a, CEP164, and IFT88 (magenta). B Immunostaining of CP110 (cyan) in control 
and patient fibroblasts, with (+) and without (-) cilia. C Bar graph quantifying CP110 dots in control and patient fibroblasts. D Representative Western blot from control and patient fibroblast lysates after 24 hours with (+) or without (-) serum. Blots were probed for CP110 and $\alpha$-Tubulin for a loading control. Bar graph quantifying relative levels of CP110 in control and patient fibroblasts with or without serum from three independent experiments. E Molecules implicated in the removal of CP110: TTBK2 and MPP9 (magenta) are normally localized at the basal body of patient fibroblasts. F Immunostaining for four transition zone proteins (magenta) in control and patient fibroblasts, + and - cilia. TMEM67, RPGRIP1L and NPHP1 are not properly localized to the base of the cilium in patient fibroblasts, while TCTN1 shows normal localization. In all panels, cilia/basal bodies were co-stained with $\mathrm{y}$-tubulin and acetylated tubulin (Acetyl/YTub, green). Scale bar $2 \mu \mathrm{m}$. G Representative immunofluorescence images showing cilia (Htr6-GFP, green) in control or CEP162 shRNA targeted cells (mCherry, magenta) as well as CEP162 shRNA targeted cells co-expressing either full-length FLAG-CEP162 or FLAG-CEP162-E646R ${ }^{\star} 5$. Scale bars, $10 \mu \mathrm{m}$. H Bar graph showing percent cilia in Control or CEP162 shRNA expressing IMCD3 cells, which were rescued by expression of the full-length FLAG-CEP162, but not FLAG-CEP162E646R ${ }^{\star} 5 .{ }^{*} p<0.016,{ }^{* *} p<0.0019$.

\section{FIGURE 6}

\section{CEP162-E646R ${ }^{\star} 5$ mutant protein is mislocalized in mouse rod photoreceptors. A}

Cetn2-GFP transgenic mouse retinal sections stained with an anti-CEP162 (magenta) antibody show that CEP162 is localized at the photoreceptor connecting cilium, which is marked by GFP fluorescence (green). Scale bar, $10 \mu \mathrm{m}$. B High-resolution Airyscan images show that CEP162 (magenta) staining decorates the distal ends of each centriole of the basal body at the base of the connecting cilium (Cetn2-GFP, green). 
Additional Airyscan images of CEP162 counterstained with multiple photoreceptor connecting cilium markers: Acetylated Tubulin (AcTub), CEP290, CEP164, and CP110 (cyan). Scale bar, $1 \mu \mathrm{m}$. C-D Wild-type mouse rods were transfected with FLAGCEP162 (C) or FLAG-CEP162-E646R*5 (D) and Rhodopsin-mCherry (red), as a transfection marker. Sections from transfected retina were stained for FLAG (magenta) to label the recombinant protein and Centrin1 (green) to label the connecting cilium. Arrows indicate the basal body of the transfected rods. Scale bars, $5 \mu \mathrm{m}$ and $2 \mu \mathrm{m}$. 


\section{REFERENCES}

1. S. K. Verbakel et al., Non-syndromic retinitis pigmentosa. Prog Retin Eye Res 66, 157186 (2018).

2. N. Patel et al., Mutations in known disease genes account for the majority of autosomal recessive retinal dystrophies. Clin Genet 94, 554-563 (2018).

3. N. Pontikos et al., Genetic Basis of Inherited Retinal Disease in a Molecularly Characterized Cohort of More Than 3000 Families from the United Kingdom. Ophthalmology 127, 1384-1394 (2020).

4. E. M. Stone et al., Clinically Focused Molecular Investigation of 1000 Consecutive Families with Inherited Retinal Disease. Ophthalmology 124, 1314-1331 (2017).

5. N. Weisschuh et al., Genetic architecture of inherited retinal degeneration in Germany: A large cohort study from a single diagnostic center over a 9-year period. Hum Mutat 41, 1514-1527 (2020).

6. L. Whelan et al., Findings from a Genotyping Study of Over 1000 People with Inherited Retinal Disorders in Ireland. Genes (Basel) 11 (2020).

7. H. P. Scholl et al., Emerging therapies for inherited retinal degeneration. Sci Trans/ Med 8, 368rv366 (2016).

8. A. Estrada-Cuzcano, R. Roepman, F. P. Cremers, A. I. den Hollander, D. A. Mans, Nonsyndromic retinal ciliopathies: translating gene discovery into therapy. Hum Mol Genet 21, R111-124 (2012).

9. H. Y. Chen, R. A. Kelley, T. Li, A. Swaroop, Primary cilia biogenesis and associated retinal ciliopathies. Semin Cell Dev Biol 110, 70-88 (2021).

10. S. C. Goetz, K. V. Anderson, The primary cilium: a signalling centre during vertebrate development. Nat Rev Genet 11, 331-344 (2010).

11. J. F. Reiter, M. R. Leroux, Genes and molecular pathways underpinning ciliopathies. Nat Rev Mol Cell Biol 18, 533-547 (2017).

12. W. J. Wang et al., CEP162 is an axoneme-recognition protein promoting ciliary transition zone assembly at the cilia base. Nat Cell Biol 15, 591-601 (2013).

13. A. Leon, B. Omri, A. Gely, C. Klein, P. Crisanti, QN1/KIAA1009: a new essential protein for chromosome segregation and mitotic spindle assembly. Oncogene 25, 1887-1895 (2006).

14. D. K. Breslow, A. J. Holland, Mechanism and Regulation of Centriole and Cilium Biogenesis. Annu Rev Biochem 88, 691-724 (2019).

15. N. Huang et al., M-Phase Phosphoprotein 9 regulates ciliogenesis by modulating CP110-CEP97 complex localization at the mother centriole. Nat Commun 9, 4511 (2018).

16. L. Cajanek, E. A. Nigg, Cep164 triggers ciliogenesis by recruiting Tau tubulin kinase 2 to the mother centriole. Proc Natl Acad Sci U S A 111, E2841-2850 (2014).

17. T. Oda, S. Chiba, T. Nagai, K. Mizuno, Binding to Cep164, but not EB1, is essential for centriolar localization of TTBK2 and its function in ciliogenesis. Genes Cells 19, 927-940 (2014).

18. C. H. Lo et al., Phosphorylation of CEP83 by TTBK2 is necessary for cilia initiation. J Cell Biol 218, 3489-3505 (2019).

19. C. S. Cowan et al., Cell Types of the Human Retina and Its Organoids at Single-Cell Resolution. Cell 182, 1623-1640 e1634 (2020).

20. B. Neron, M. Marx, P. Crisanti, Role of QN1 protein in cell proliferation arrest and differentiation during the neuroretina development. Mech Dev 102, 107-117 (2001). 
21. H. Higginbotham, S. Bielas, T. Tanaka, J. G. Gleeson, Transgenic mouse line with greenfluorescent protein-labeled Centrin 2 allows visualization of the centrosome in living cells. Transgenic Res 13, 155-164 (2004).

22. T. Matsuda, C. L. Cepko, Controlled expression of transgenes introduced by in vivo electroporation. Proc Natl Acad Sci U S A 104, 1027-1032 (2007).

23. T. Nagase et al., Prediction of the coding sequences of unidentified human genes. XIII. The complete sequences of 100 new cDNA clones from brain which code for large proteins in vitro. DNA Res 6, 63-70 (1999).

24. A. I. den Hollander et al., Mutations in the CEP290 (NPHP6) gene are a frequent cause of Leber congenital amaurosis. Am J Hum Genet 79, 556-561 (2006).

25. M. J. Landrum et al., ClinVar: public archive of interpretations of clinically relevant variants. Nucleic Acids Res 44, D862-868 (2016).

26. P. D. Stenson et al., The Human Gene Mutation Database (HGMD((R))): optimizing its use in a clinical diagnostic or research setting. Hum Genet 139, 1197-1207 (2020).

27. A. N. Abou Tayoun et al., Recommendations for interpreting the loss of function PVS1 ACMG/AMP variant criterion. Hum Mutat 39, 1517-1524 (2018).

28. S. Ellard et al., ACGS Best Practice Guidelines for Variant Classification. Assoc Clin Genet Sci (2019).

29. K. Nykamp et al., Sherloc: a comprehensive refinement of the ACMG-AMP variant classification criteria. Genet Med 19, 1105-1117 (2017).

30. S. Richards et al., Standards and guidelines for the interpretation of sequence variants: a joint consensus recommendation of the American College of Medical Genetics and Genomics and the Association for Molecular Pathology. Genet Med 17, 405-424 (2015).

31. M. N. Preising et al., Biallelic mutation of human SLC6A6 encoding the taurine transporter TAUT is linked to early retinal degeneration. FASEB J 33, 11507-11527 (2019).

32. A. Kawalia et al., Leveraging the power of high performance computing for next generation sequencing data analysis: tricks and twists from a high throughput exome workflow. PLoS One 10, e0126321 (2015).

33. B. B. Beck et al., Mutation of POC1B in a severe syndromic retinal ciliopathy. Hum Mutat 35, 1153-1162 (2014).

34. S. M. Elsayed et al., Non-manifesting AHI1 truncations indicate localized loss-offunction tolerance in a severe Mendelian disease gene. Hum Mol Genet 24, 2594-2603 (2015).

35. M. Lek et al., Analysis of protein-coding genetic variation in 60,706 humans. Nature 536, 285-291 (2016).

36. V. Plagnol et al., A robust model for read count data in exome sequencing experiments and implications for copy number variant calling. Bioinformatics 28, 2747-2754 (2012).

37. D. Seelow, M. Schuelke, F. Hildebrandt, P. Nurnberg, HomozygosityMapper--an interactive approach to homozygosity mapping. Nucleic Acids Res 37, W593-599 (2009).

38. S. Purcell et al., PLINK: a tool set for whole-genome association and population-based linkage analyses. Am J Hum Genet 81, 559-575 (2007).

39. T. Sante et al., ViVar: a comprehensive platform for the analysis and visualization of structural genomic variation. PLoS One 9, e113800 (2014).

40. M. Quinodoz et al., AutoMap is a high performance homozygosity mapping tool using next-generation sequencing data. Nat Commun 12, 518 (2021). 
41. F. A. Wolf, P. Angerer, F. J. Theis, SCANPY: large-scale single-cell gene expression data analysis. Genome Biol 19, 15 (2018).

42. M. Vangipuram, D. Ting, S. Kim, R. Diaz, B. Schule, Skin punch biopsy explant culture for derivation of primary human fibroblasts. J Vis Exp 10.3791/3779, e3779 (2013).

43. F. Hauser et al., A non-invasive diagnostic assay for rapid detection and characterization of aberrant mRNA-splicing by nonsense mediated decay inhibition. Mol Genet Metab 130, 27-35 (2020).

44. J. N. Pearring, W. J. Spencer, E. C. Lieu, V. Y. Arshavsky, Guanylate cyclase 1 relies on rhodopsin for intracellular stability and ciliary trafficking. Elife 4 (2015).

45. R. Y. Salinas et al., Photoreceptor discs form through peripherin-dependent suppression of ciliary ectosome release. J Cell Biol 216, 1489-1499 (2017).

46. G. J. Pazour et al., The intraflagellar transport protein, IFT88, is essential for vertebrate photoreceptor assembly and maintenance. J Cell Biol 157, 103-113 (2002).

47. S. Finkelstein et al., Phosphoinositide Profile of the Mouse Retina. Cells 9 (2020).

48. D. H. Hong, G. Yue, M. Adamian, T. Li, Retinitis pigmentosa GTPase regulator (RPGRr)interacting protein is stably associated with the photoreceptor ciliary axoneme and anchors RPGR to the connecting cilium. J Biol Chem 276, 12091-12099 (2001).

49. M. A. Robichaux et al., Defining the layers of a sensory cilium with STORM and cryoelectron nanoscopy. Proc Natl Acad Sci U S A 116, 23562-23572 (2019). 


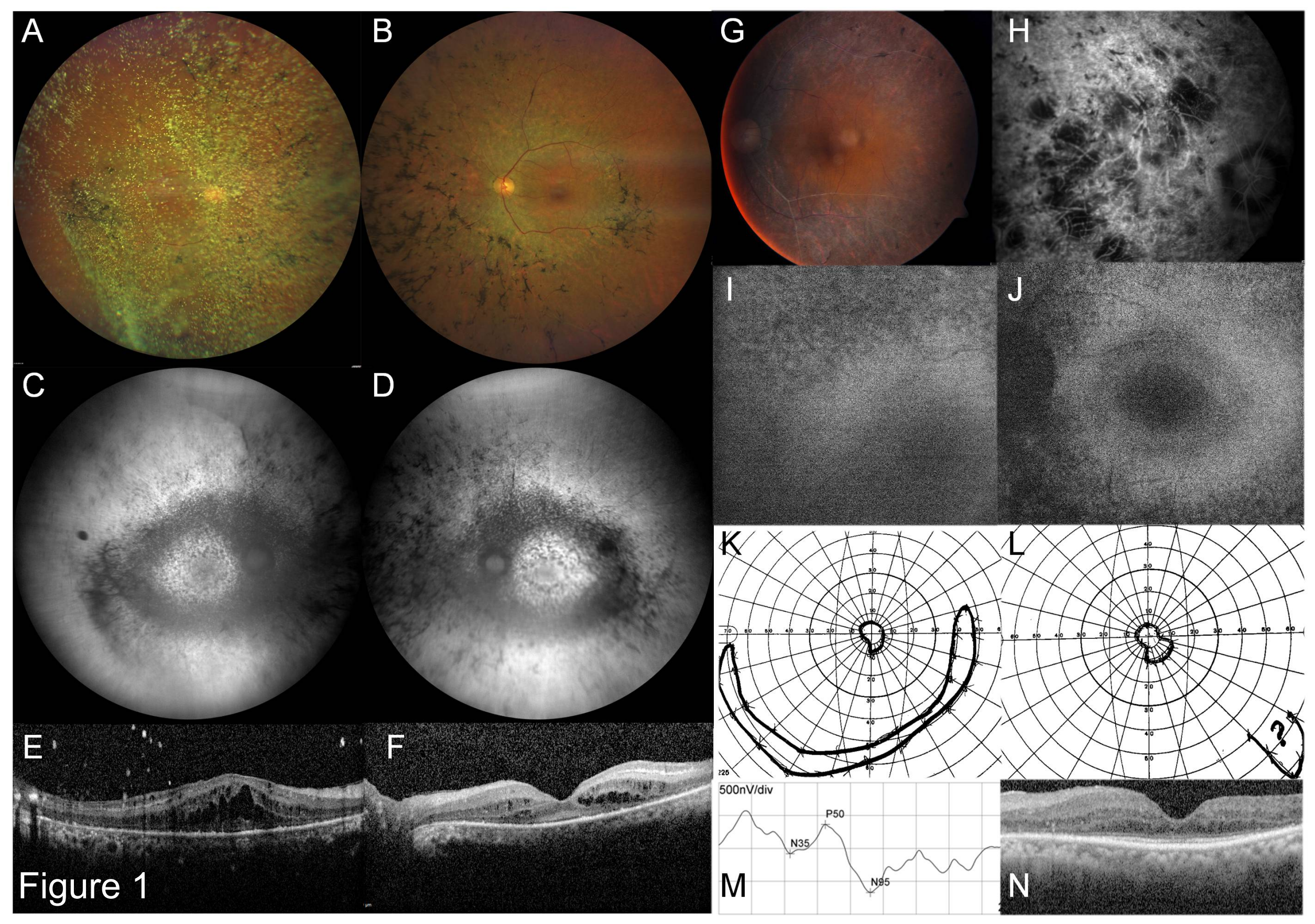




\section{CEP162}

$\mathbf{N}$

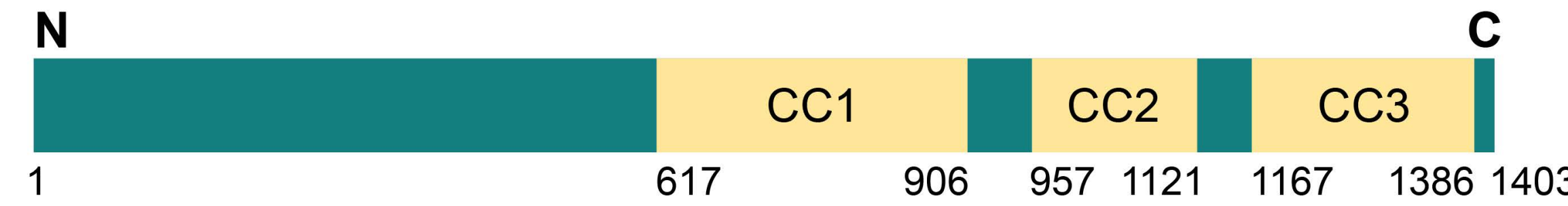

CEP162-E646R*5

$\mathrm{N}$

1

C

FLAG-CEP162
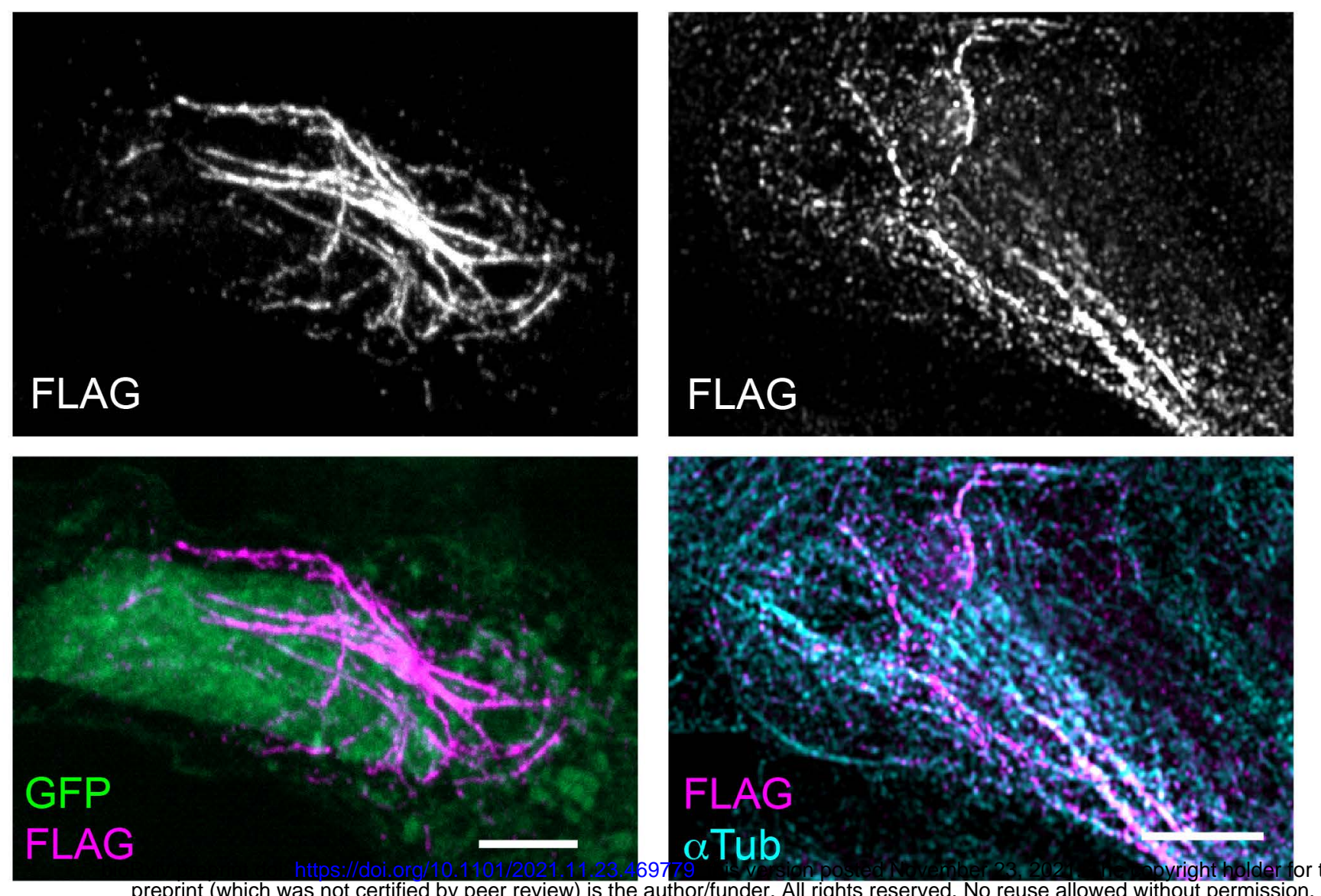

FLAG-CEP162-E646R*5

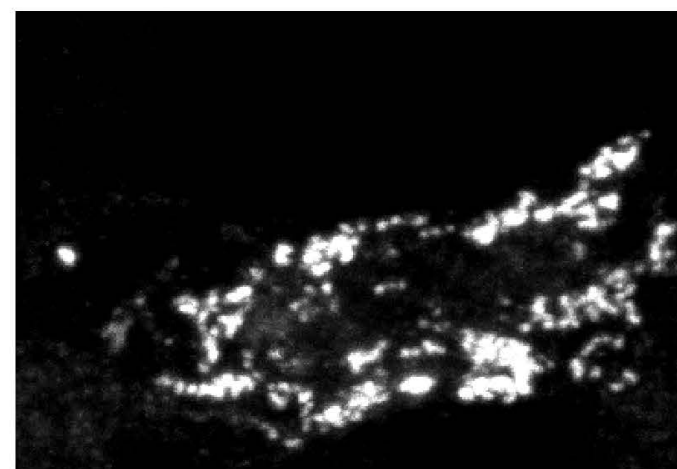

FLAG
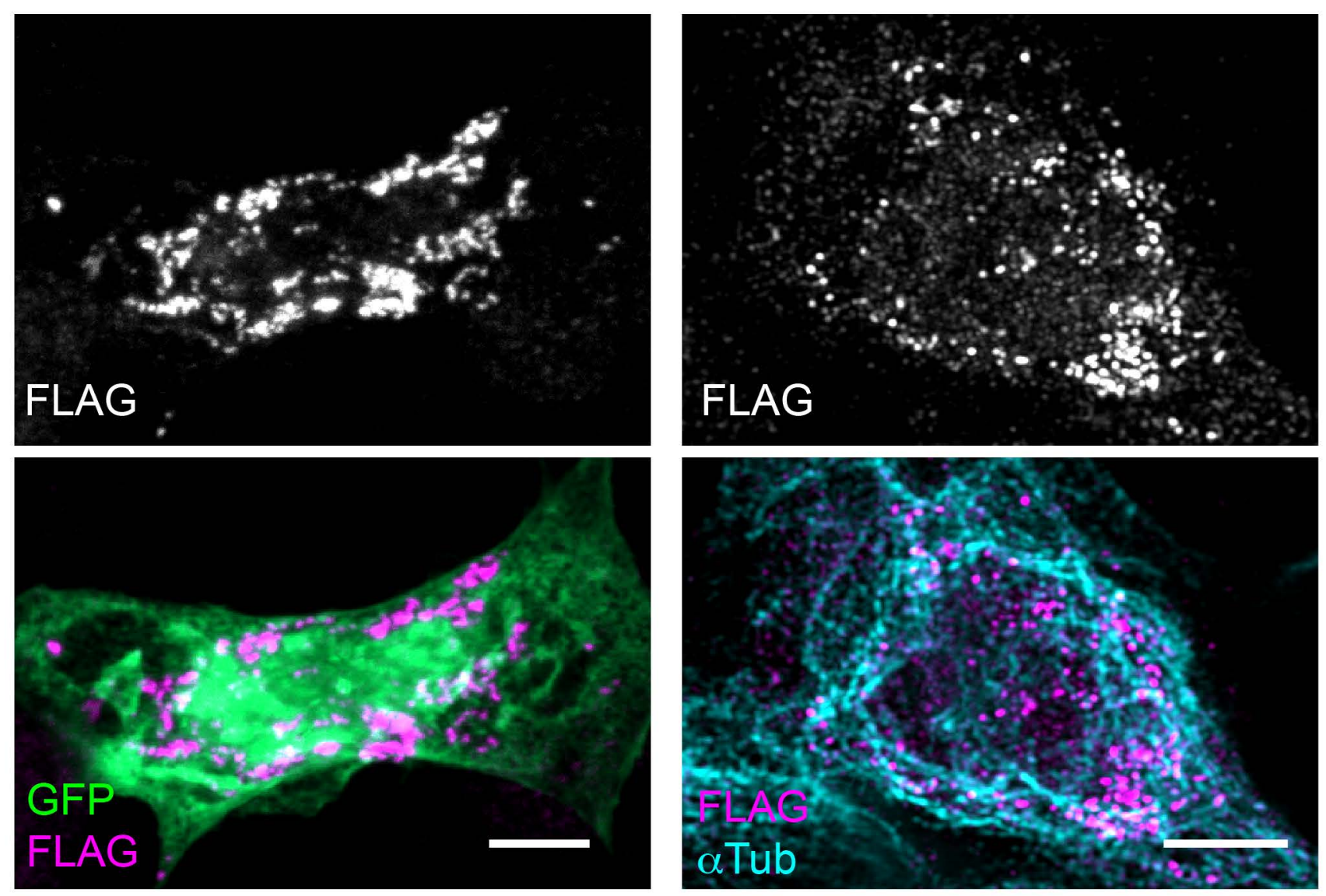

E

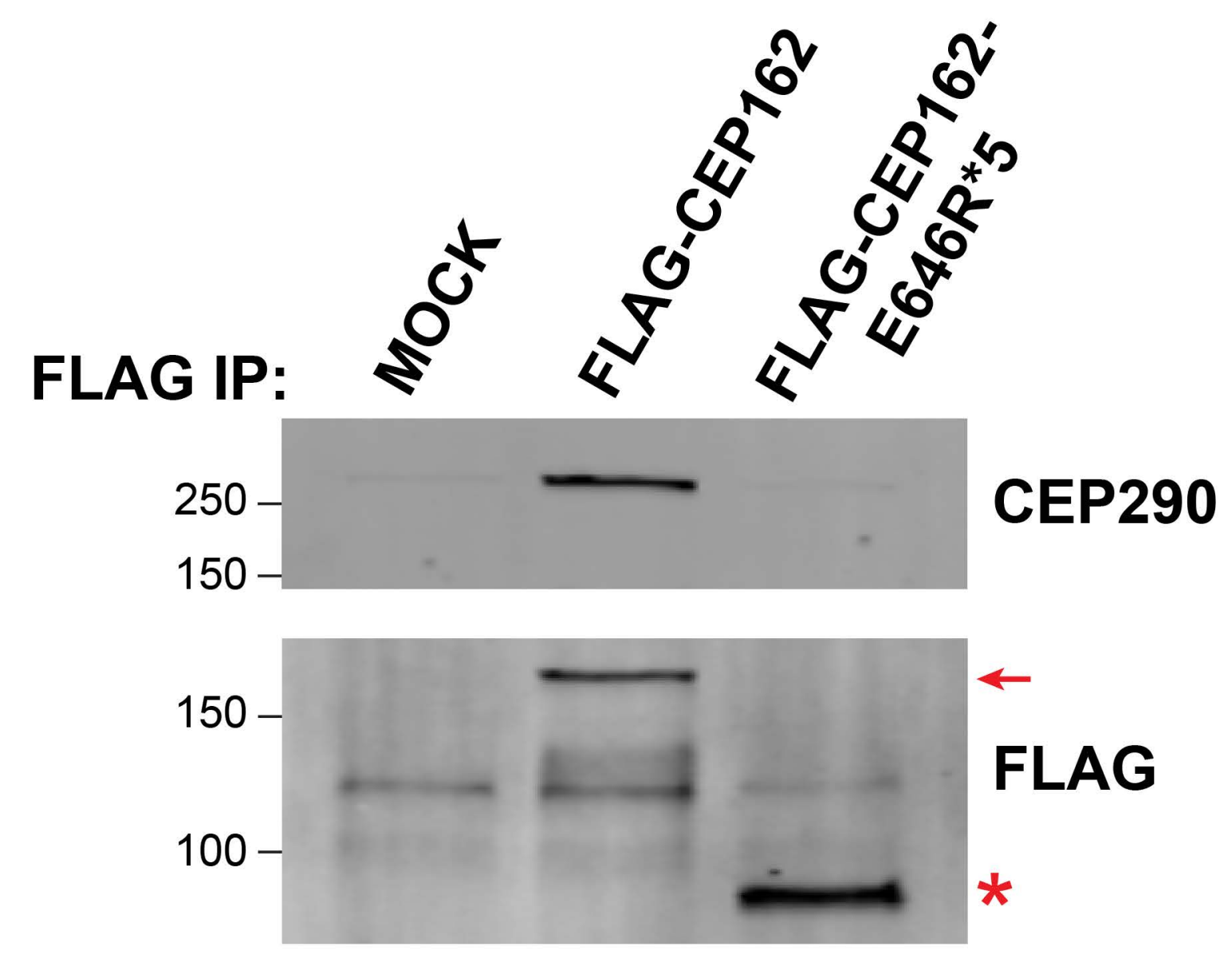

Input (5\%):

Figure 3

D
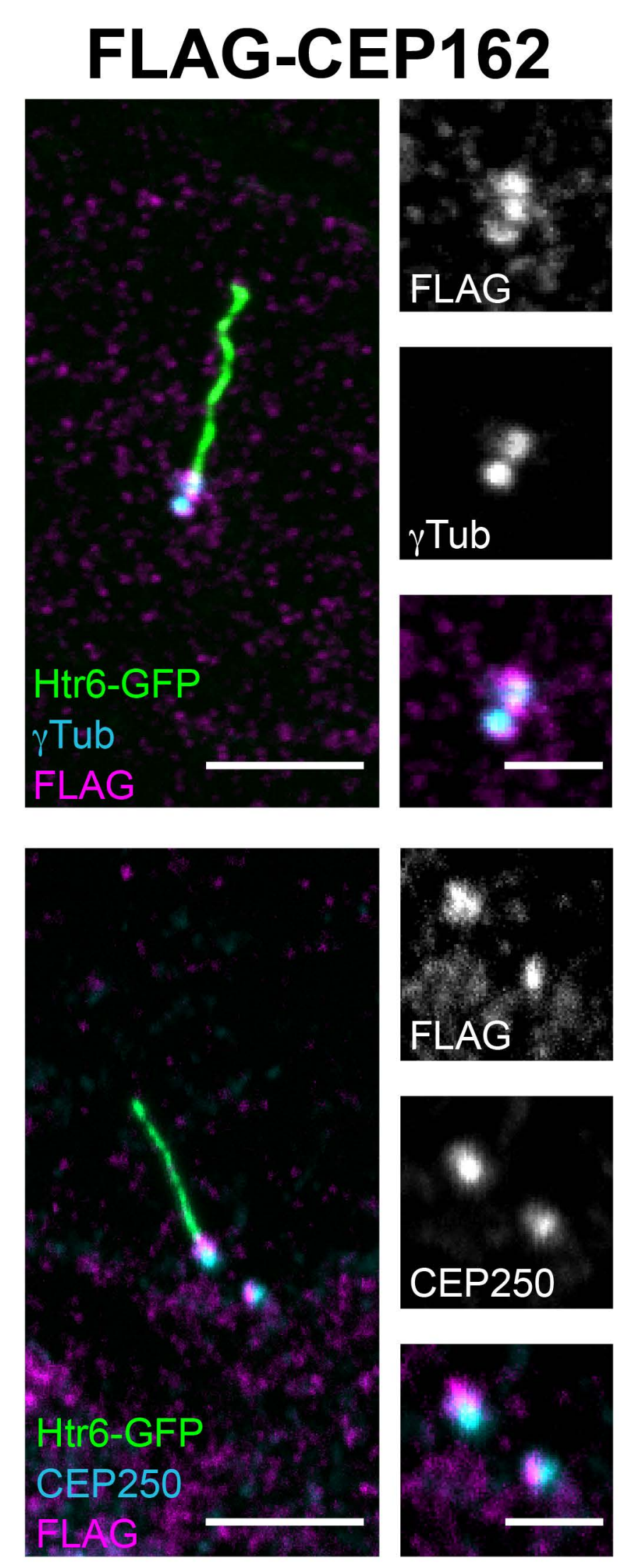

$\mathbf{F}$

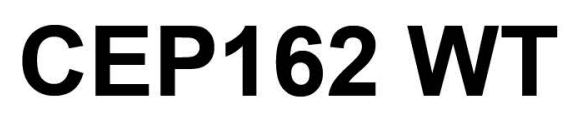
Control Fibroblast
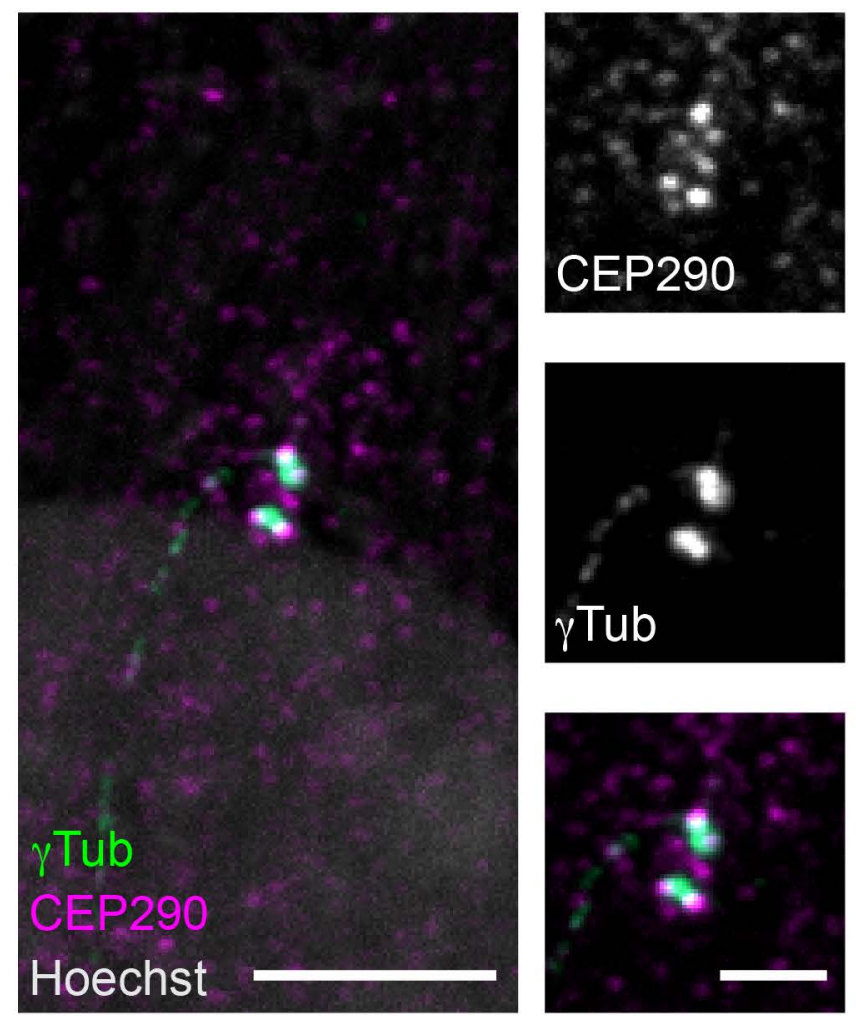
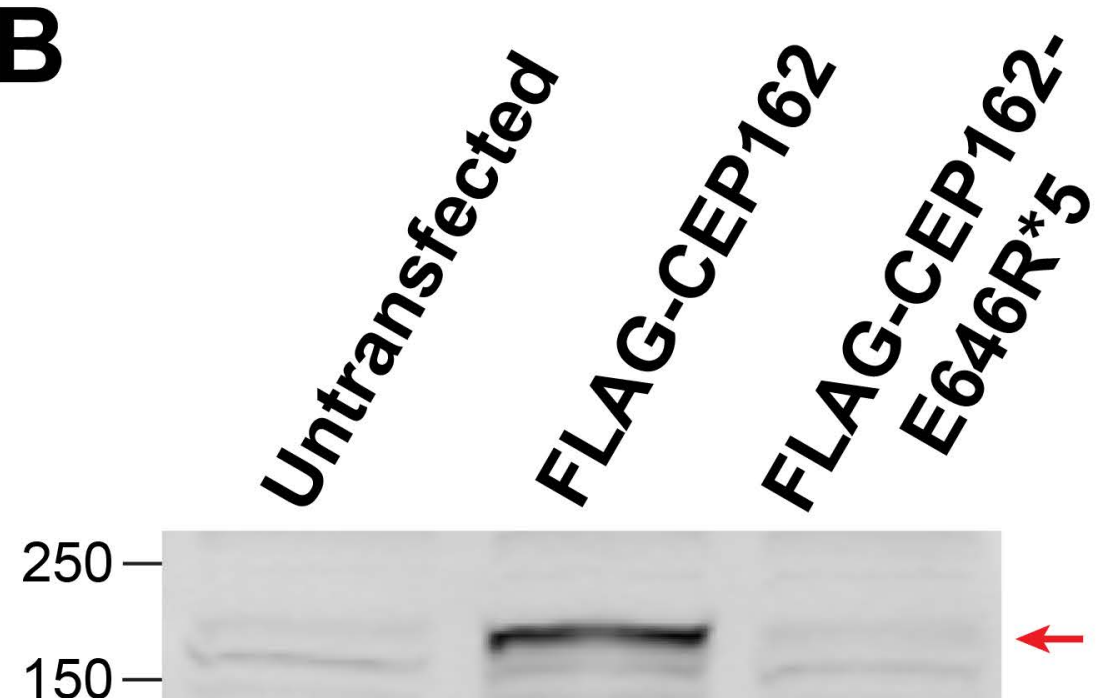

$\mathrm{N}$-Terminal CEP162

*

$\alpha$-Tubulin

FLAG-CEP162E646R*5
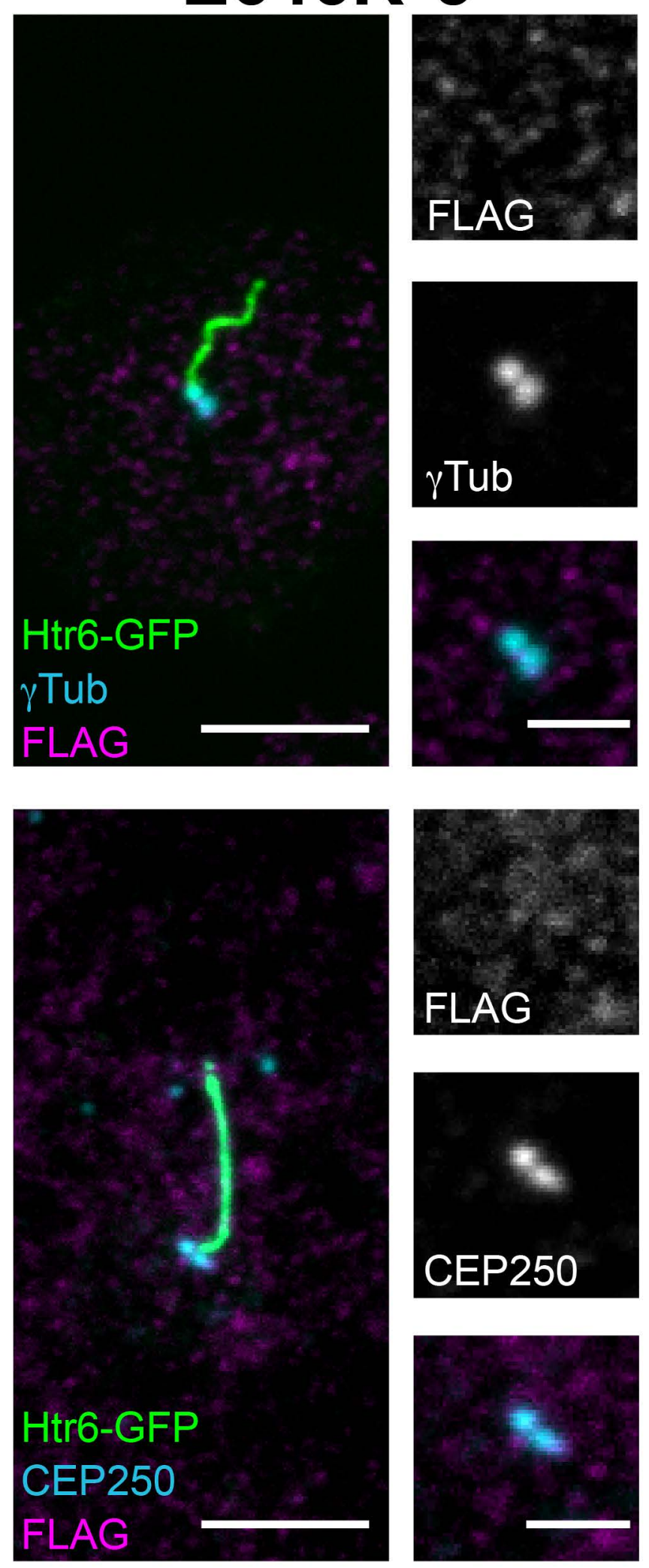

CEP162-E646R*5 Patient Fibroblast
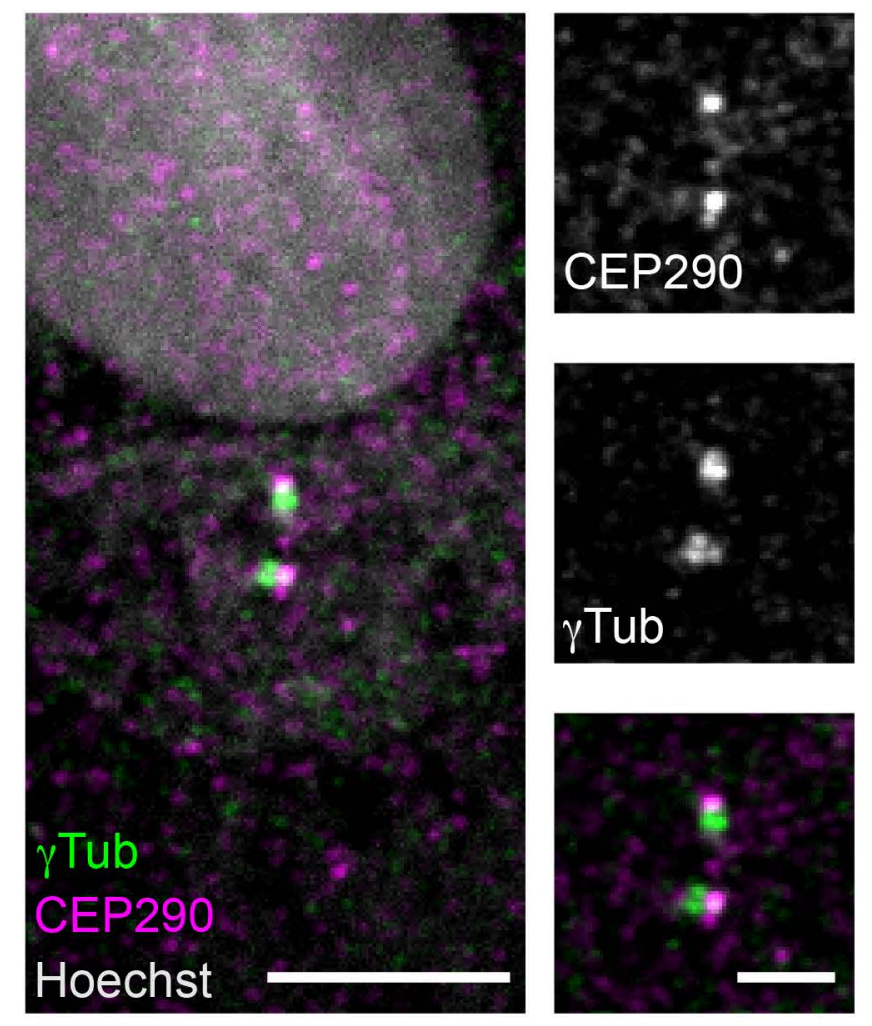
A Control CEP162 WT

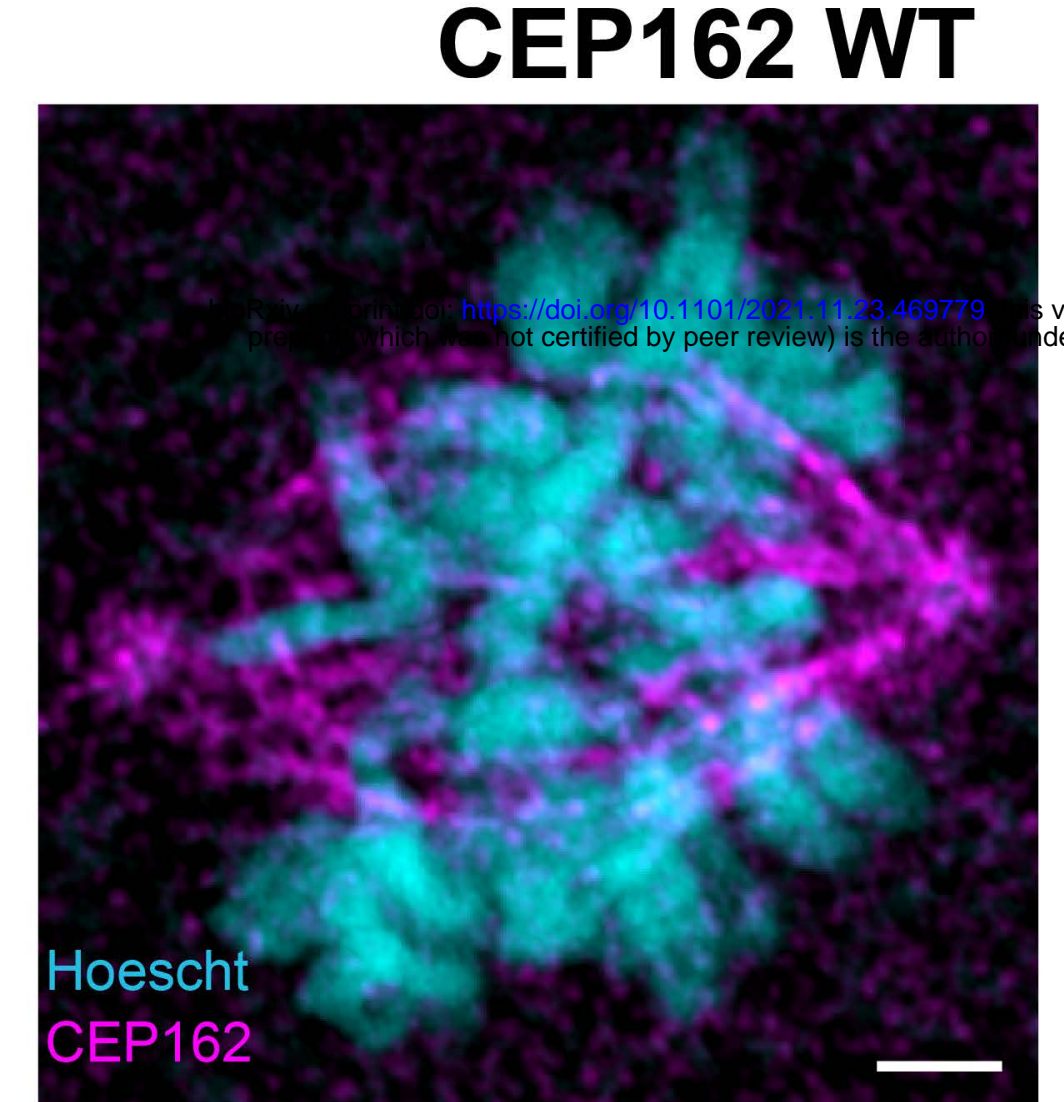

$\mathbf{F}$
Patient CEP162-E646R*5

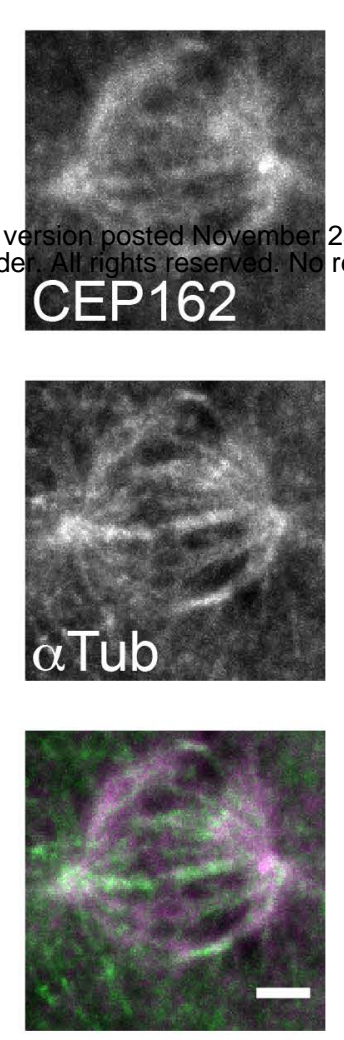

EP162

Patient CEP162-E646R*5

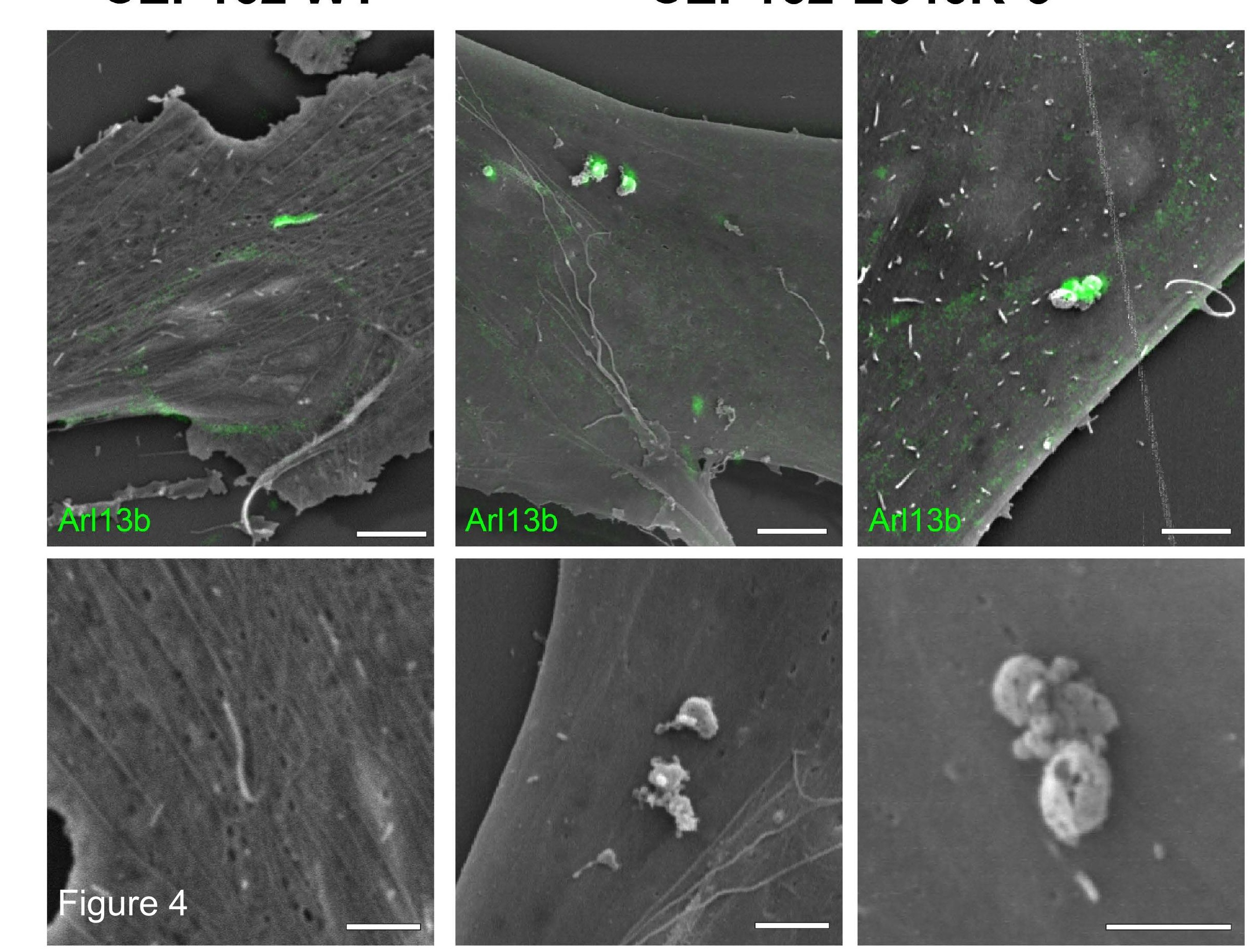

Patient

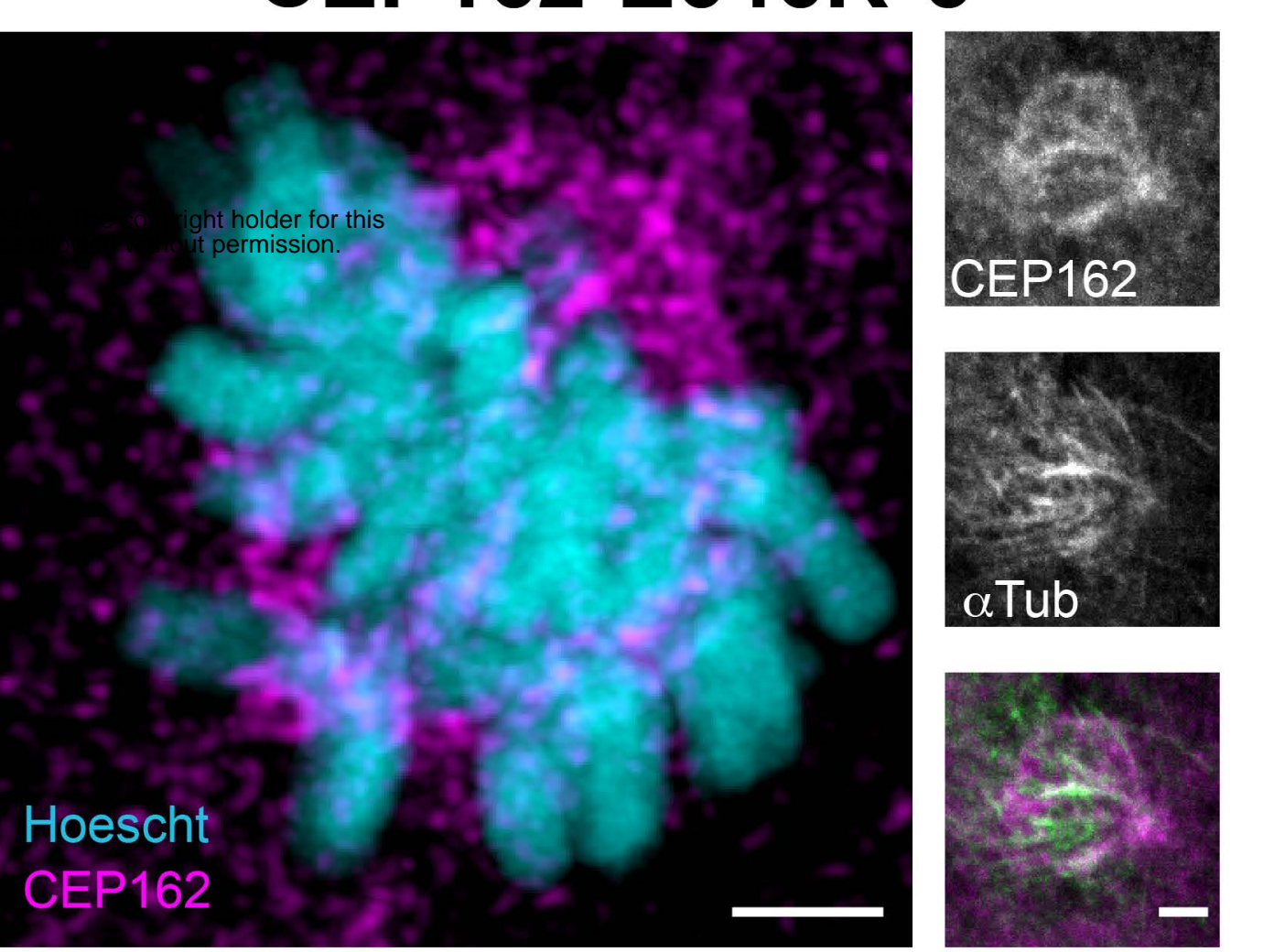

2.

Thub
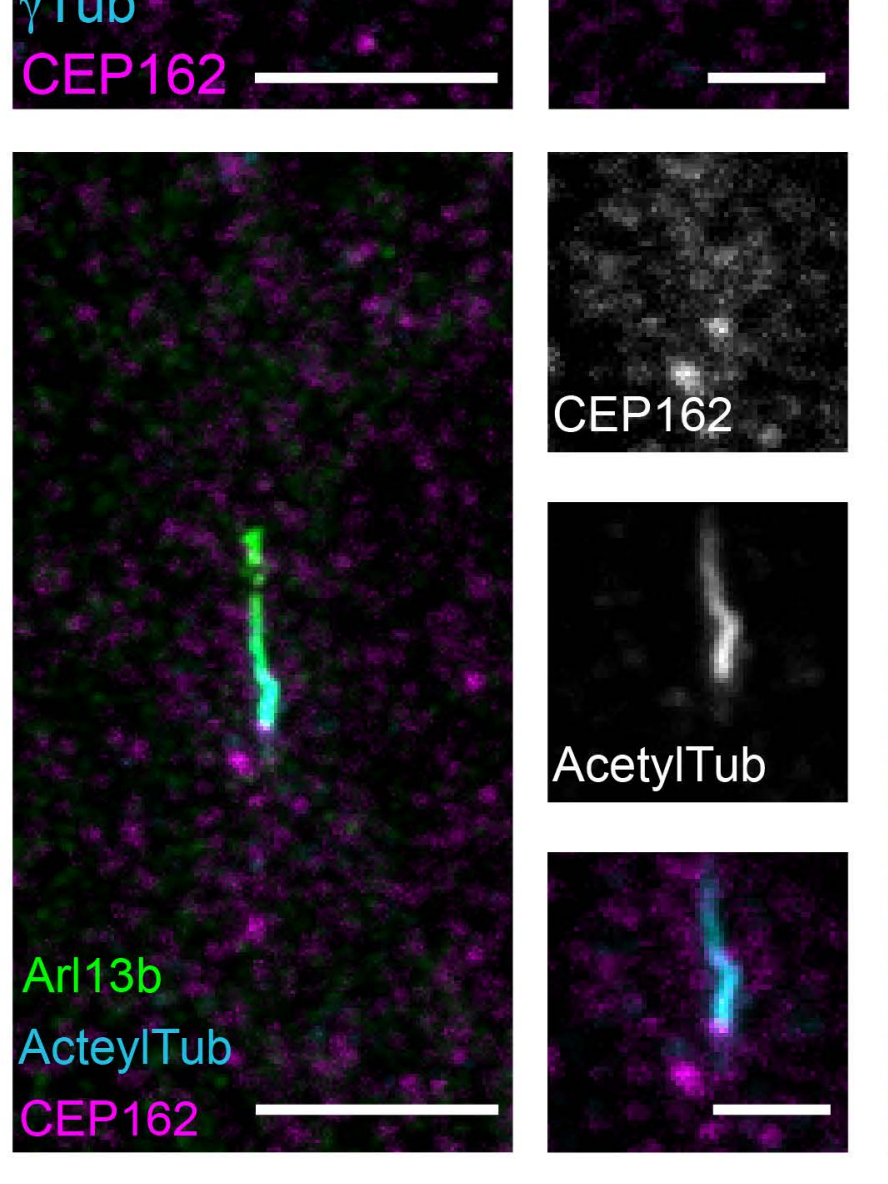

E

Control CEP162 WT

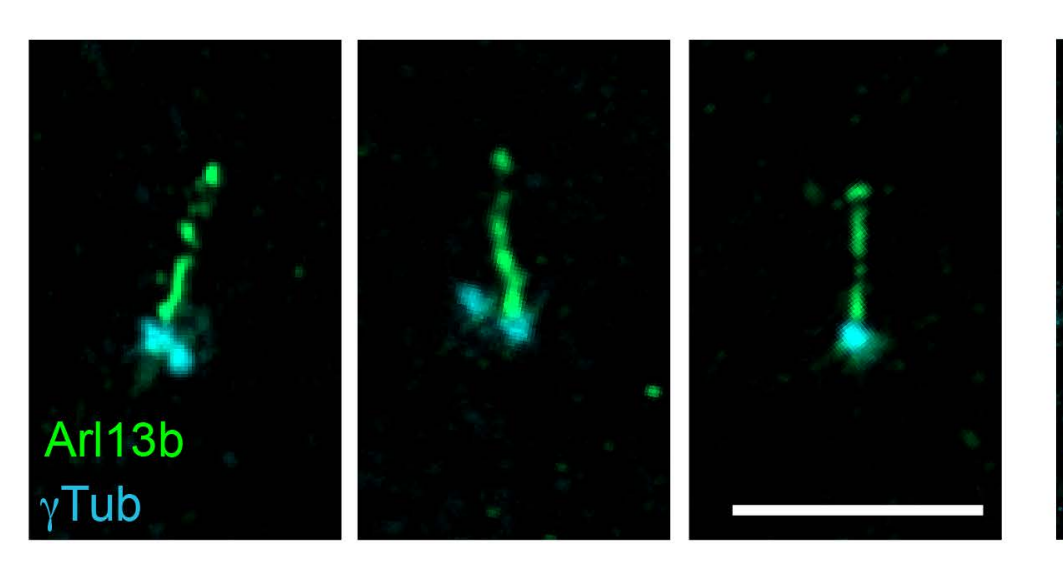

CEP162-E646R*5
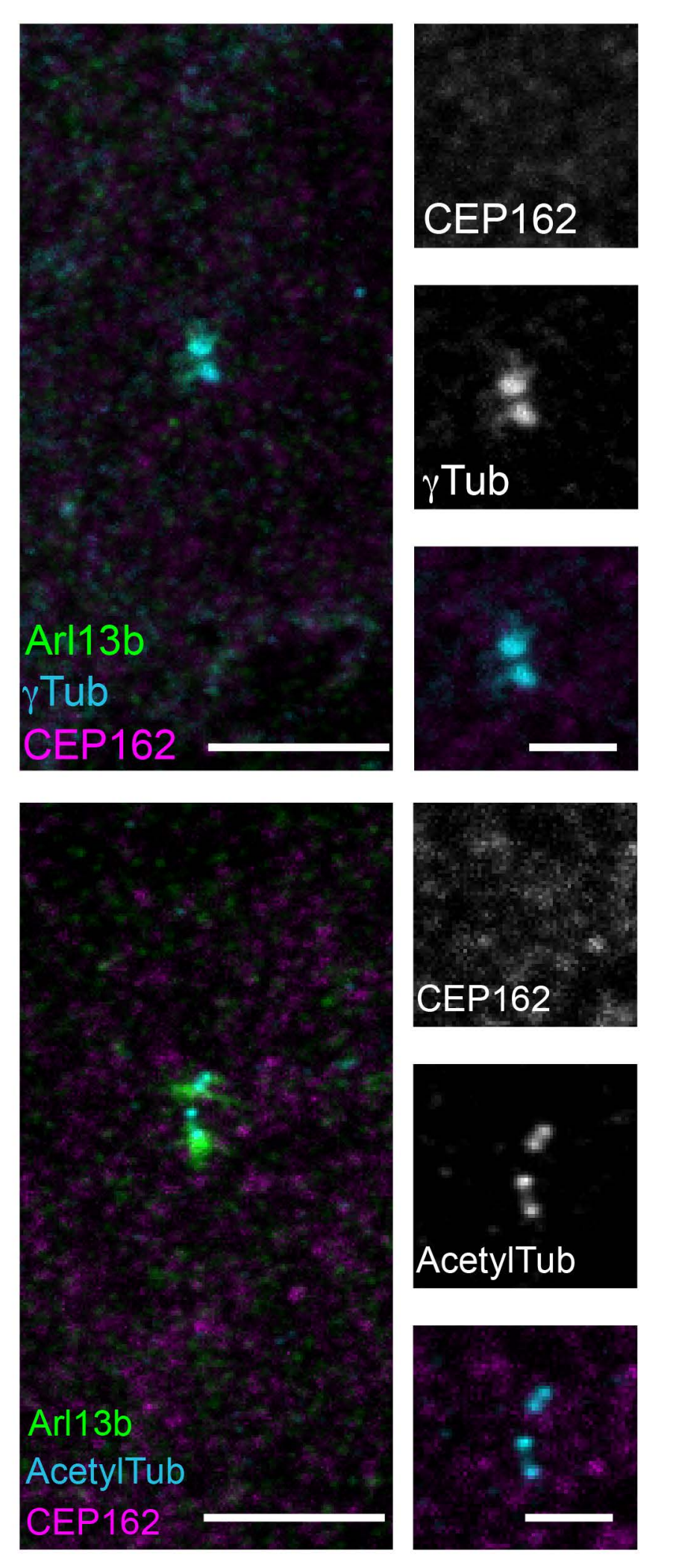

Patient CEP162-E646R*5

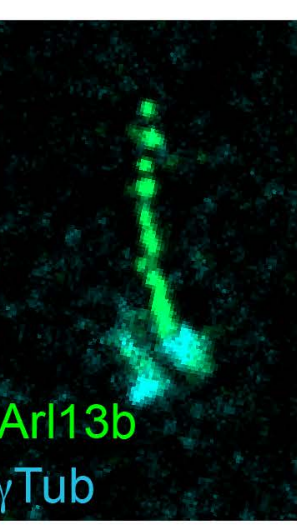

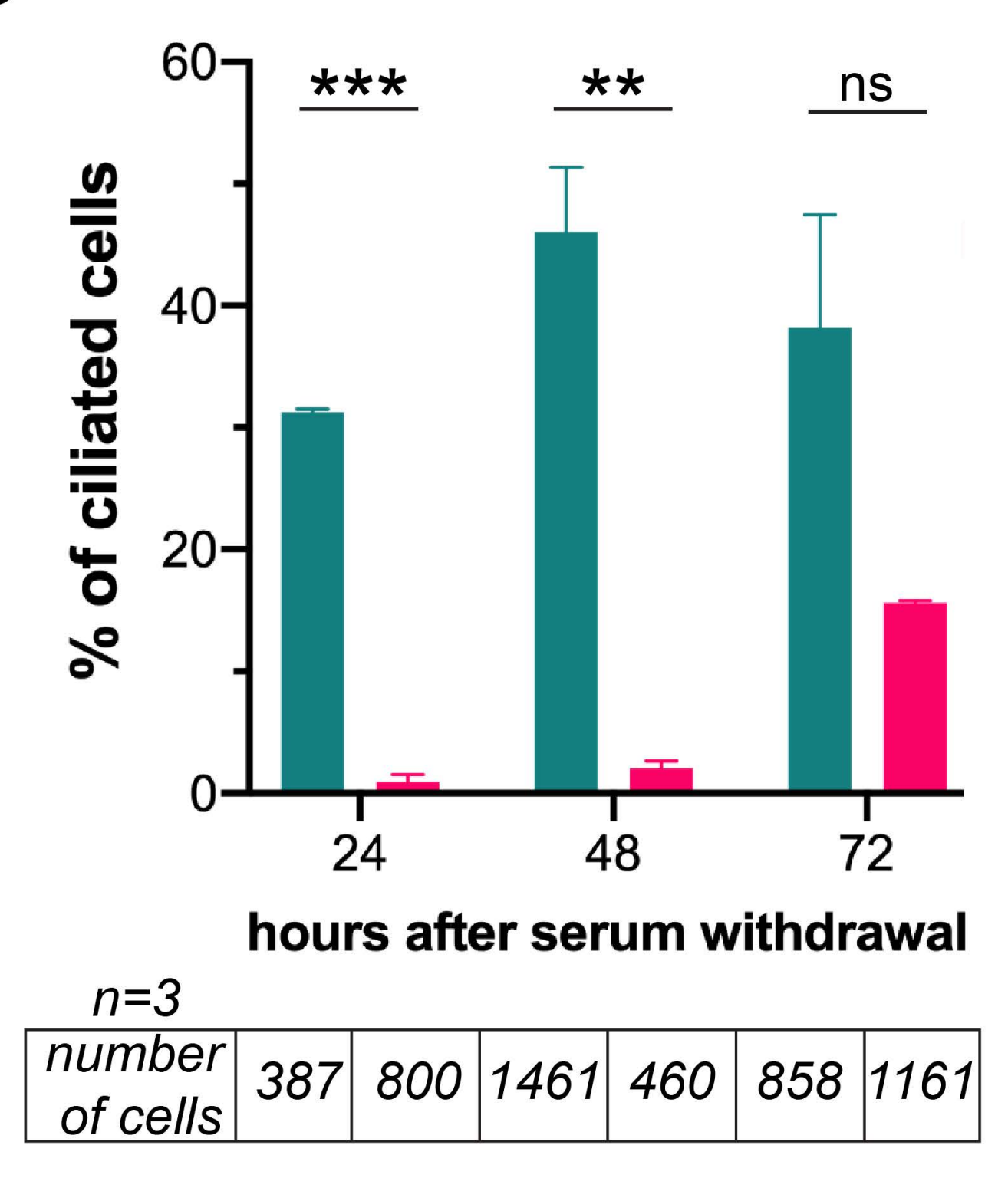

D
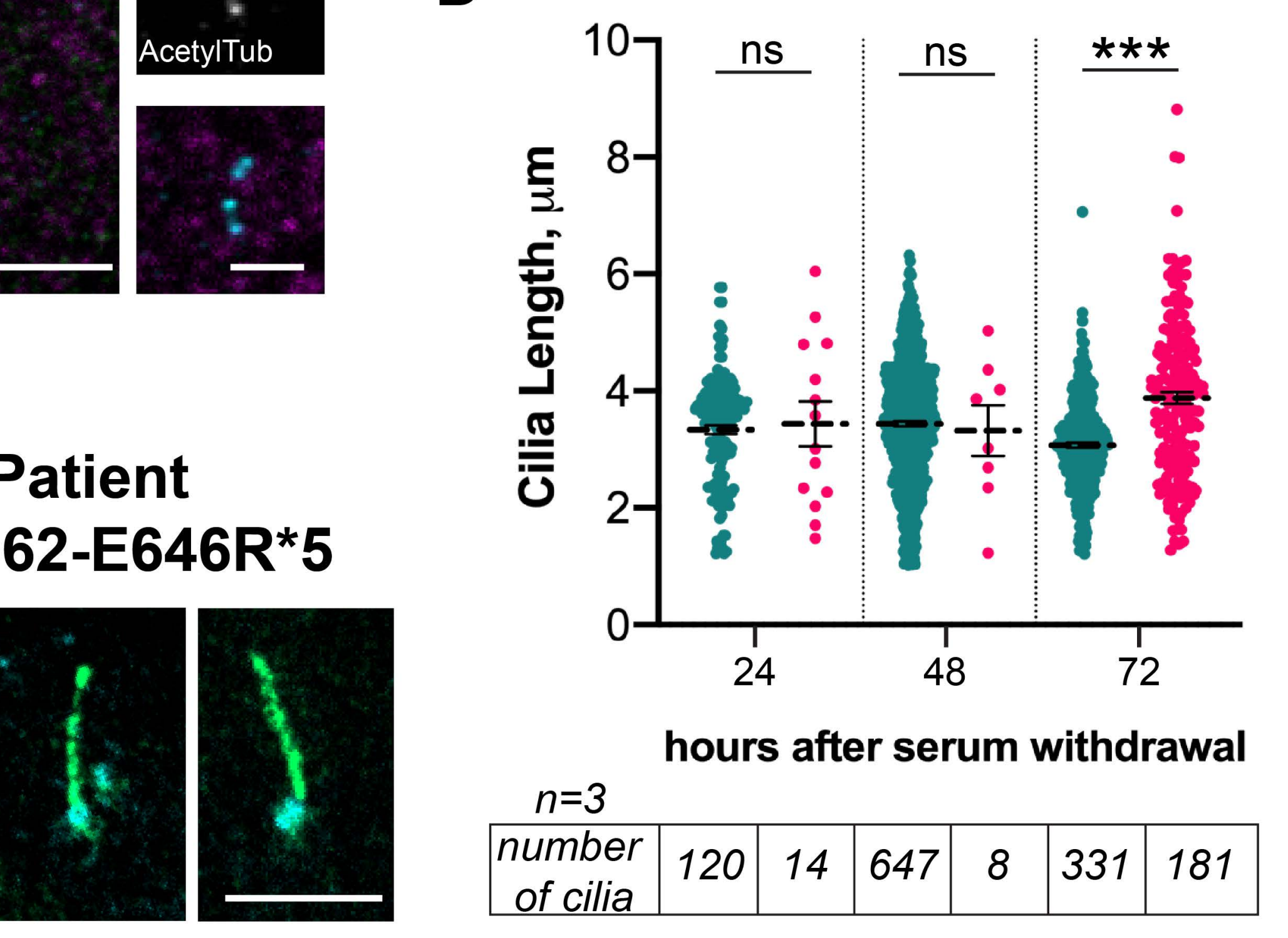

hours after serum withdrawal 
$\begin{array}{ccc}\text { A } & \begin{array}{c}\text { CEP162 WT } \\ \text { Control Fibroblast }\end{array} & \begin{array}{c}\text { CEP162-E646R } \\ \text { Patient Fibroblast }\end{array} \\ & \text { Patient }\end{array}$

cilis
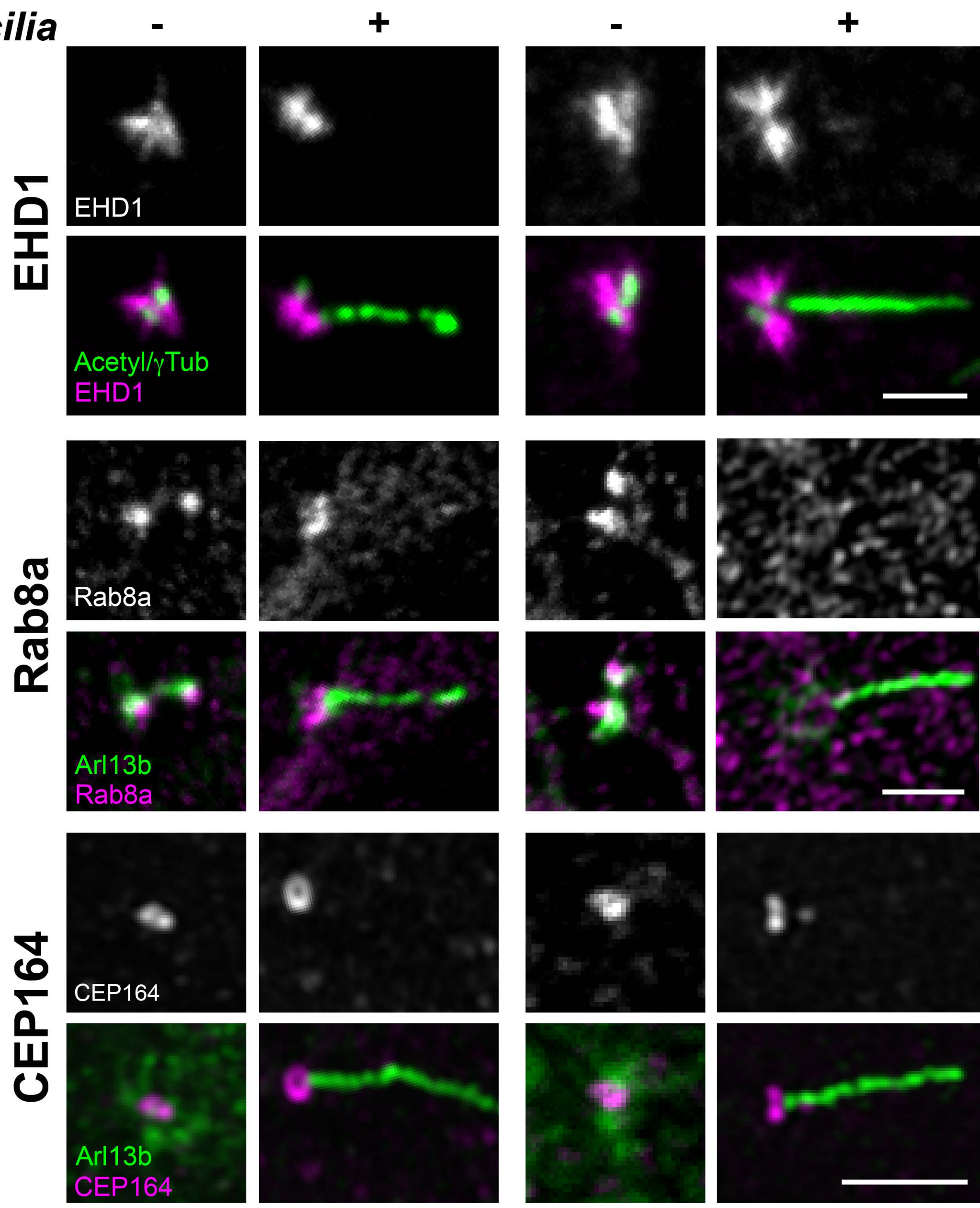

\begin{tabular}{ll}
$\infty$ & $\infty$ \\
$\infty$ & IFT88 \\
\hline
\end{tabular}

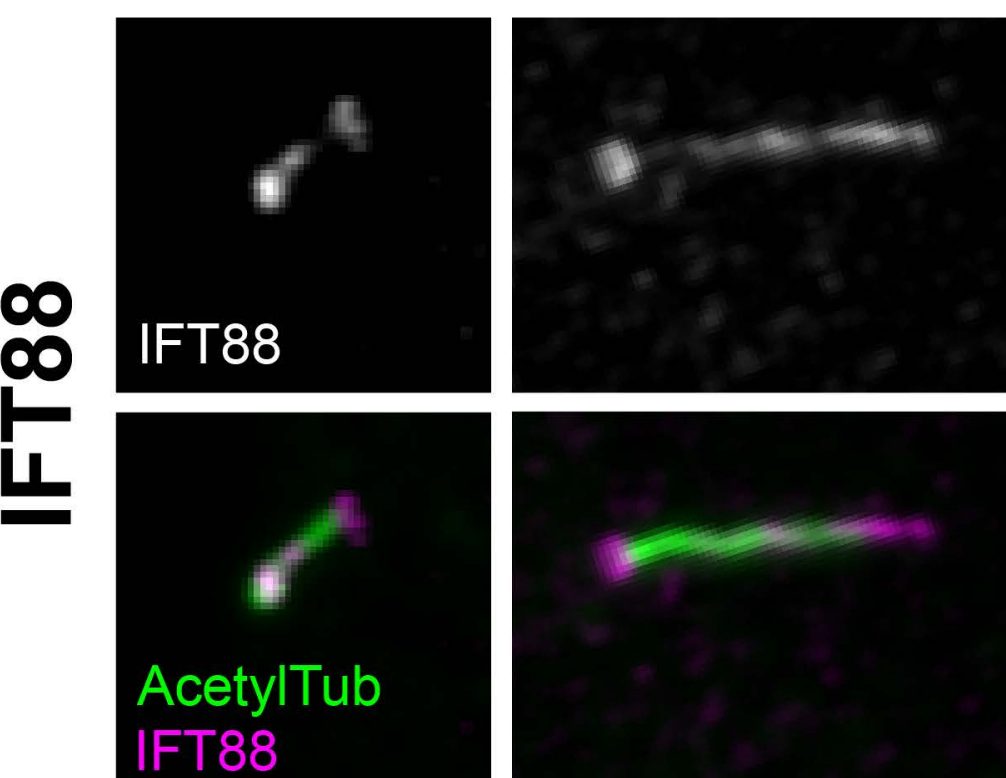

$\mathbf{F}$

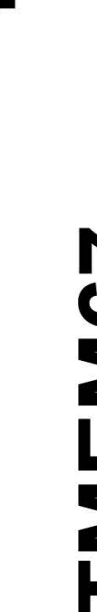

$\sum_{1}^{0}$

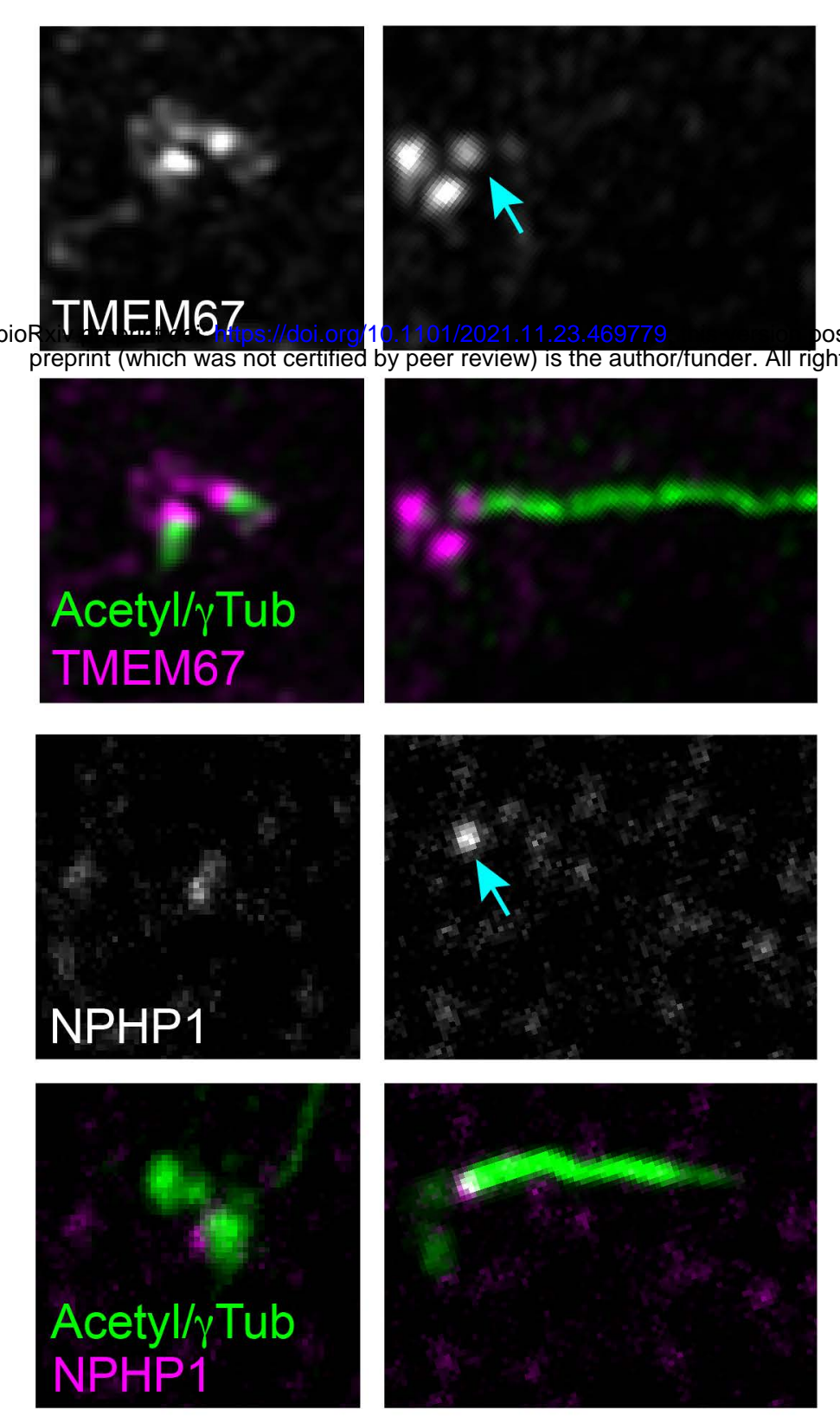

뭉
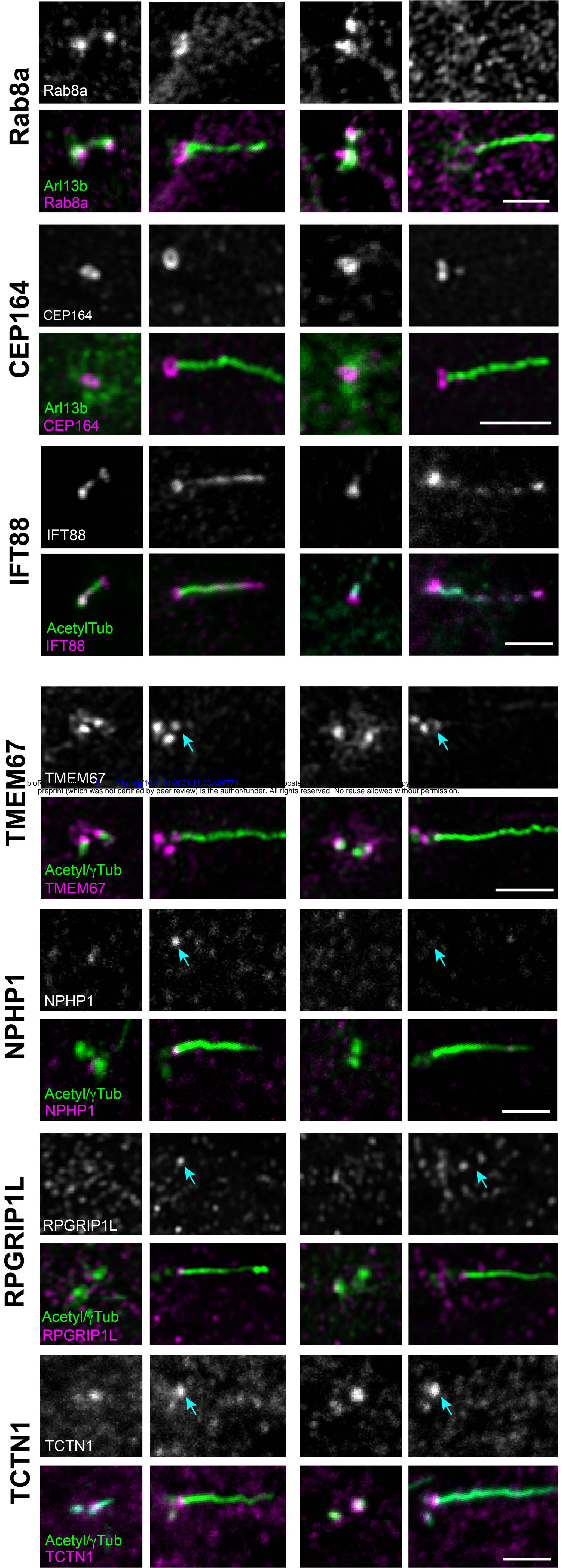

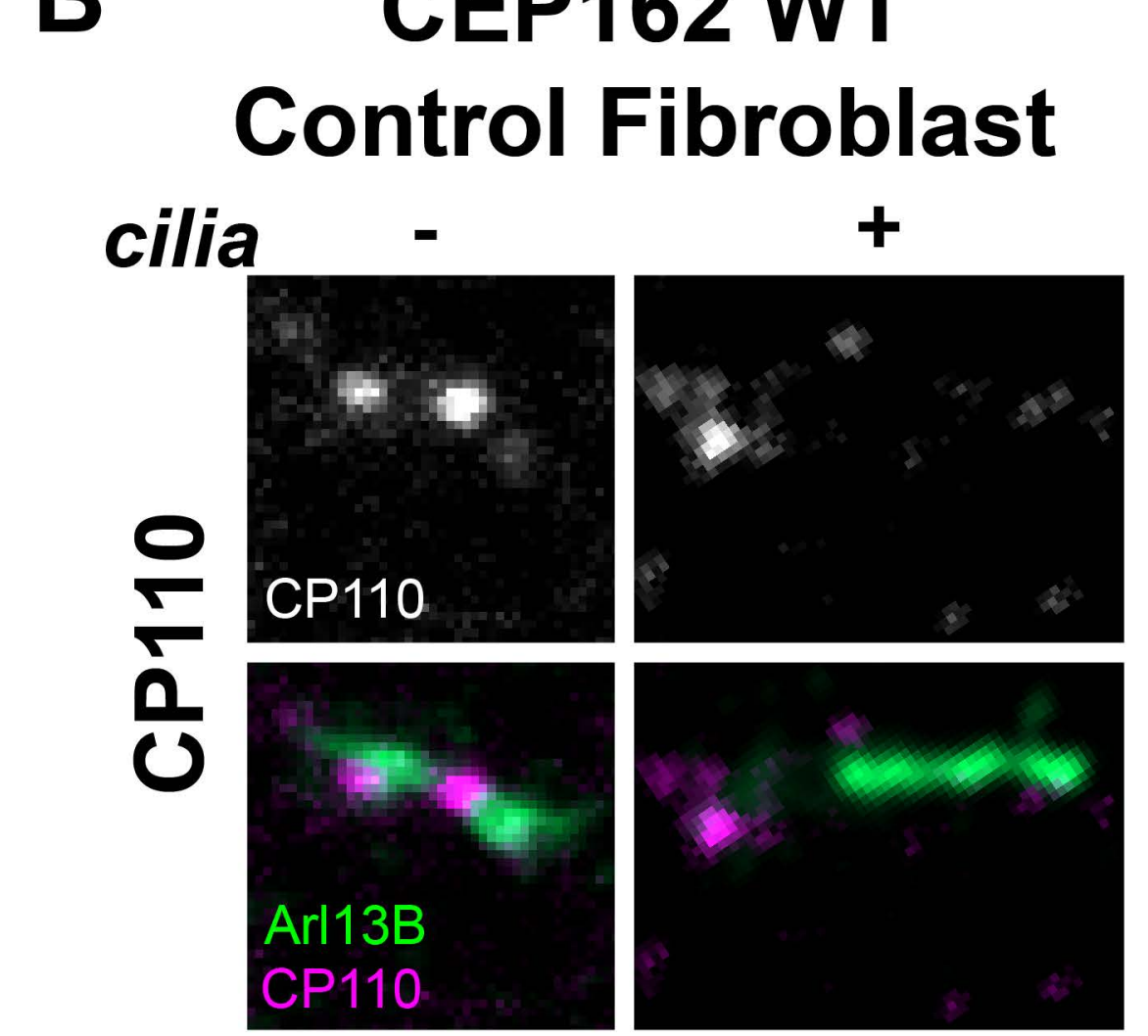

Patient Fibroblast

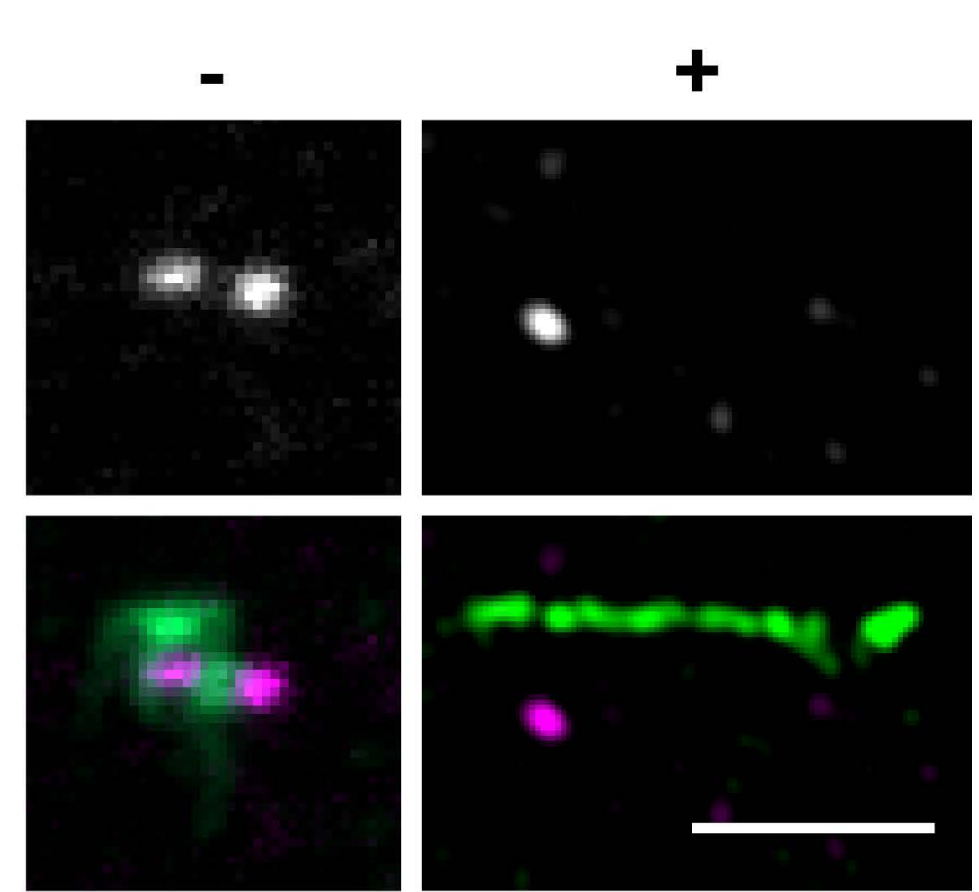

D
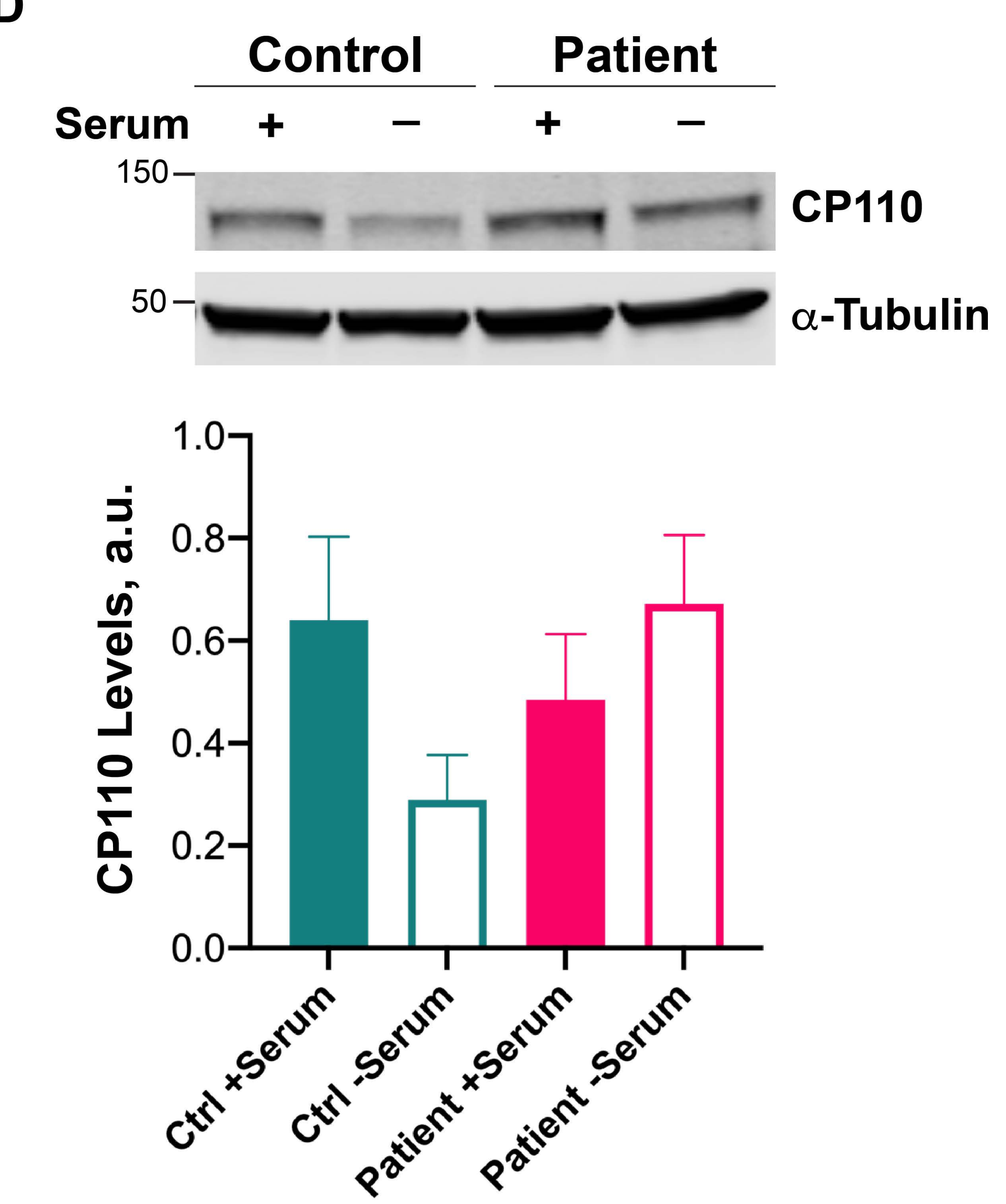

G

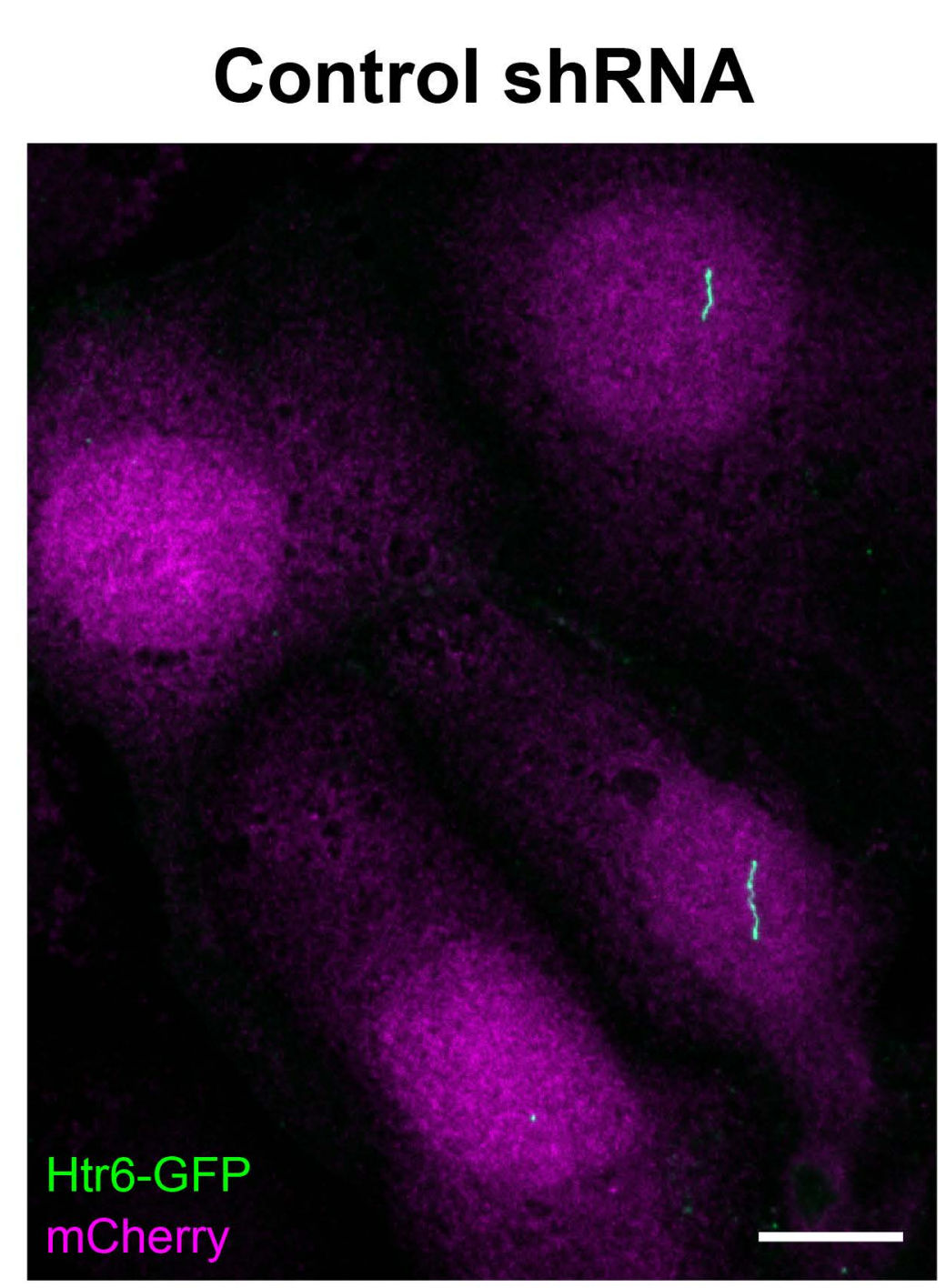

CEP162 shRNA FLAG-CEP162

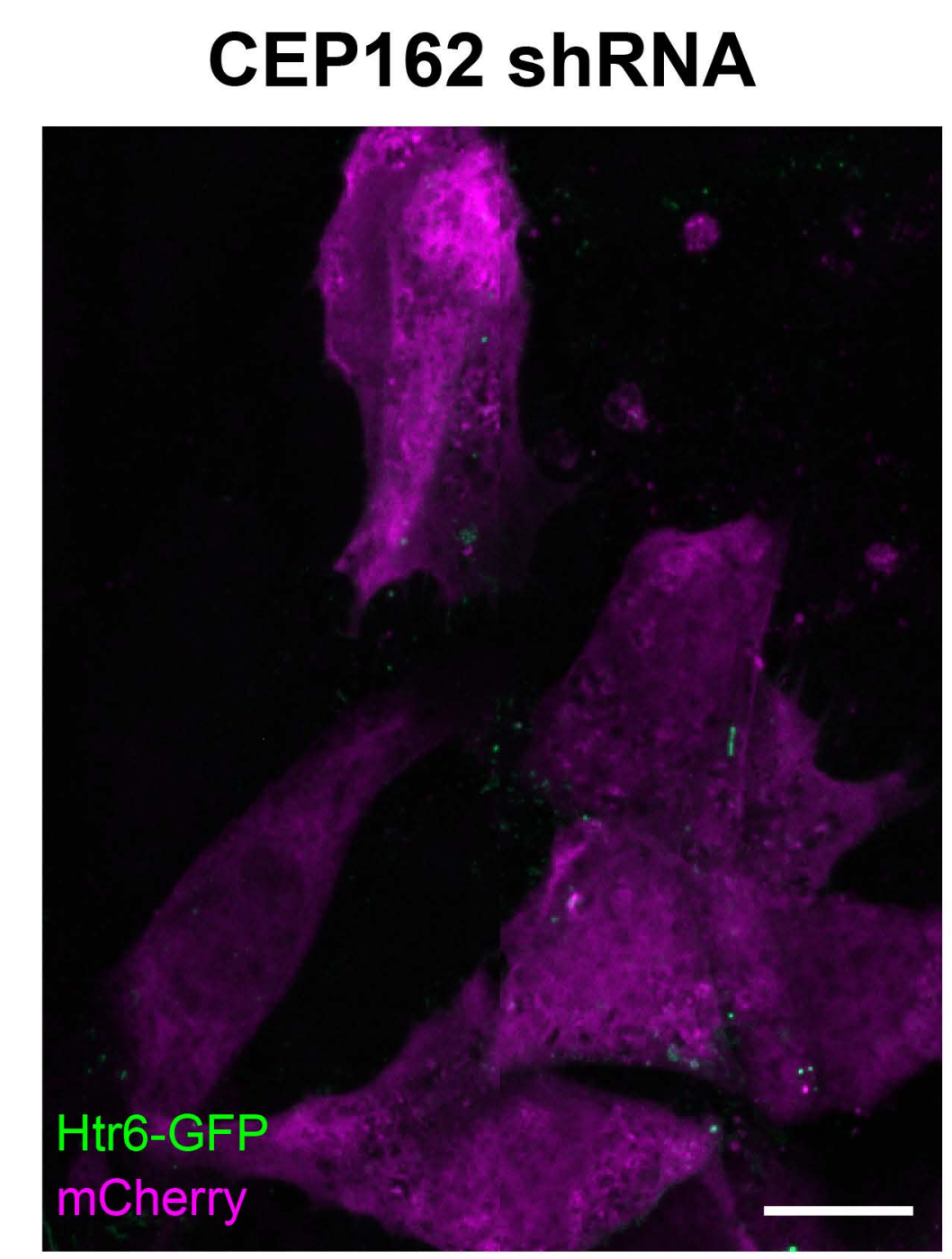

CEP162 shRNA FLAG-CEP162-E646R5

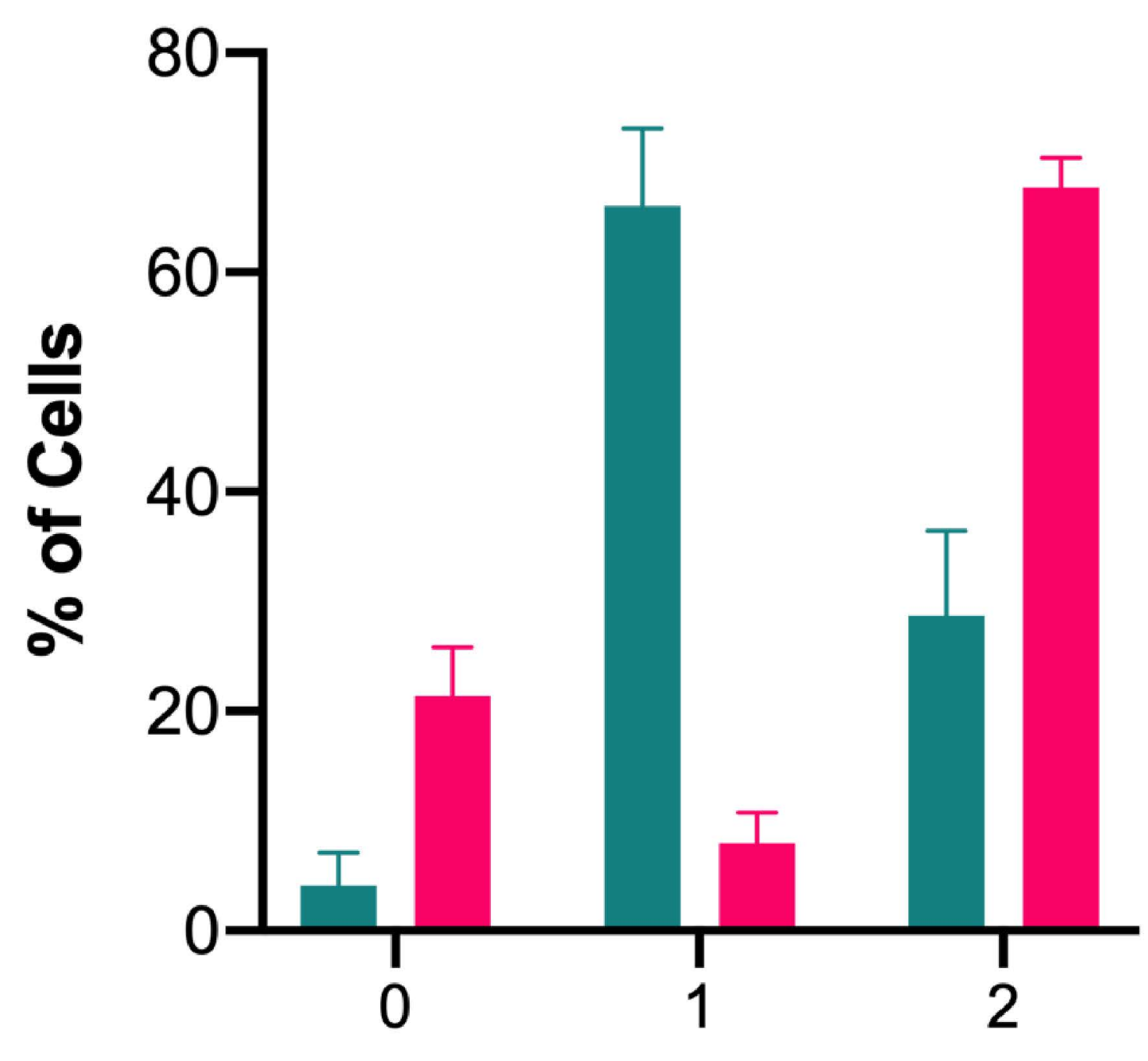

Control

CEP162 WT

Patient

CEP162-E646R5
E Control Fibroblast cilia
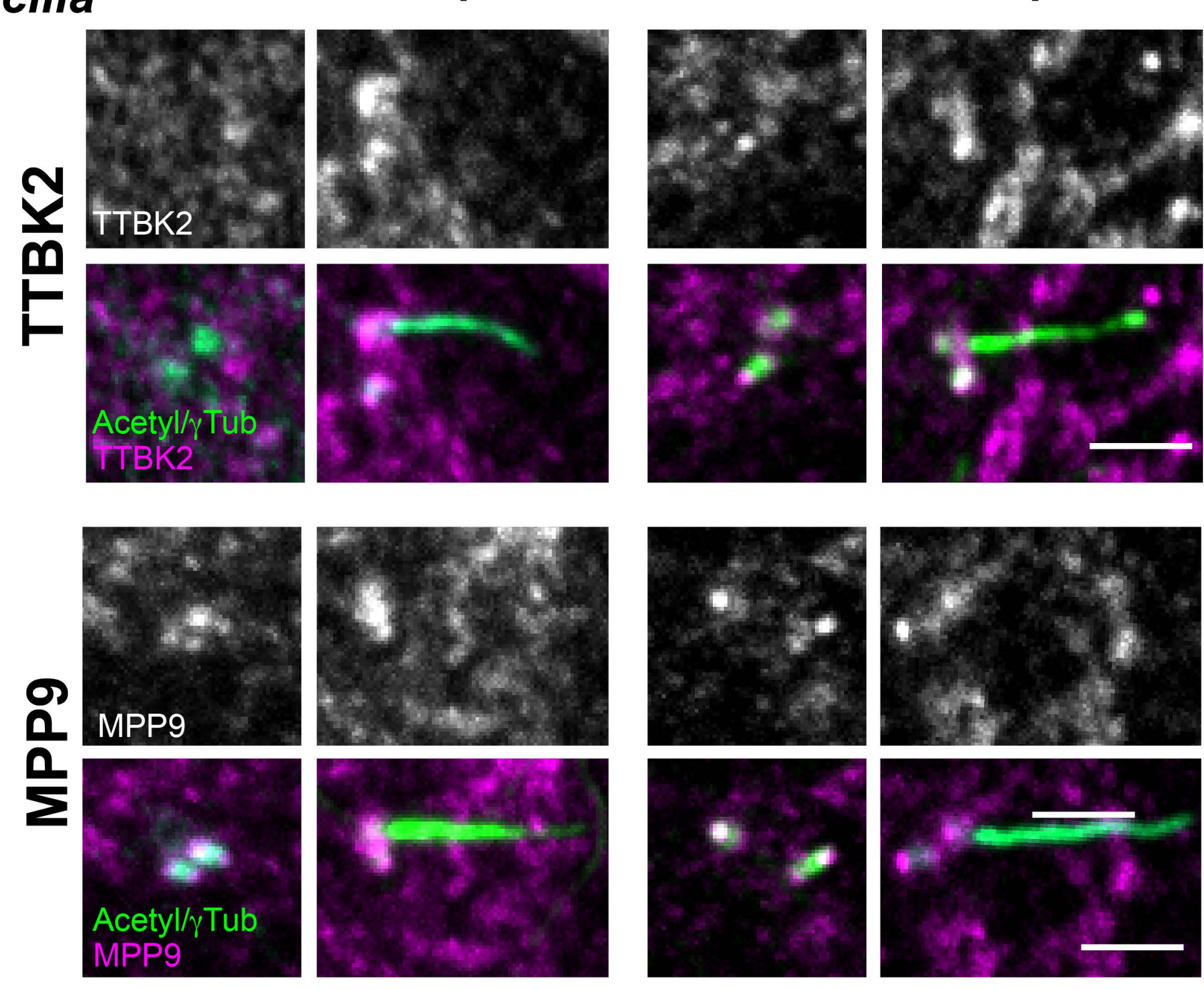

H

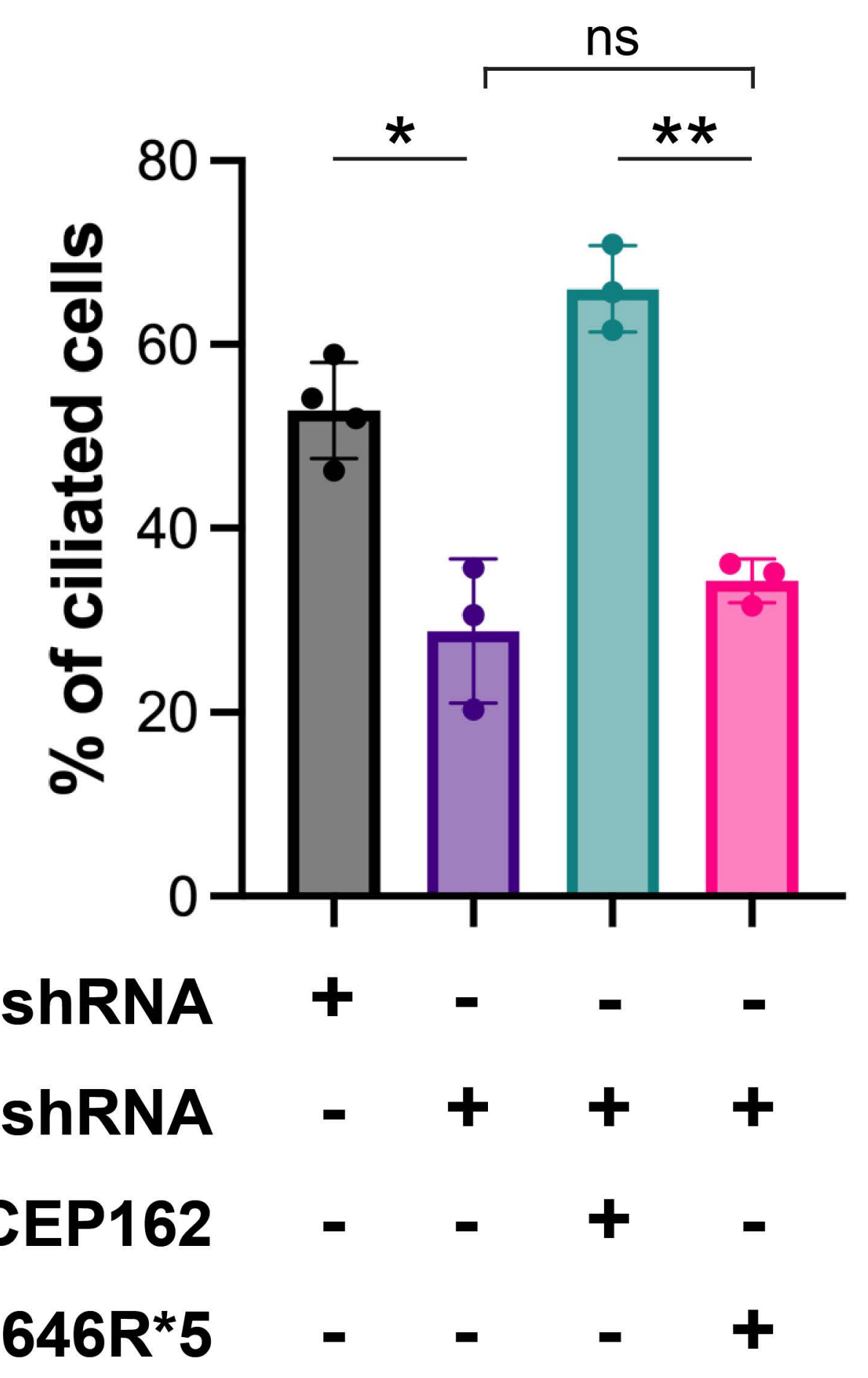


